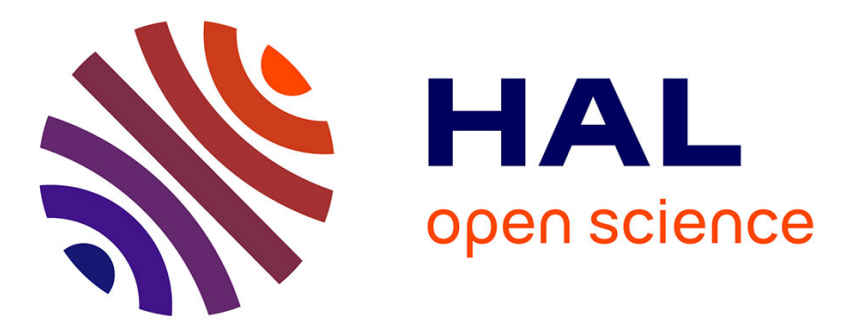

\title{
Reverse engineering reusable software components from object-oriented APIs
}

Anas Shatnawi, Abdelhak-Djamel Seriai, Houari Sahraoui, Zakarea Al-Shara

\section{To cite this version:}

Anas Shatnawi, Abdelhak-Djamel Seriai, Houari Sahraoui, Zakarea Al-Shara. Reverse engineering reusable software components from object-oriented APIs. Journal of Systems and Software, 2017, 131, pp.442-460. 10.1016/j.jss.2016.06.101 . lirmm-01932852

\section{HAL Id: lirmm-01932852 \\ https://hal-lirmm.ccsd.cnrs.fr/lirmm-01932852}

Submitted on 23 Nov 2018

HAL is a multi-disciplinary open access archive for the deposit and dissemination of scientific research documents, whether they are published or not. The documents may come from teaching and research institutions in France or abroad, or from public or private research centers.
L'archive ouverte pluridisciplinaire HAL, est destinée au dépôt et à la diffusion de documents scientifiques de niveau recherche, publiés ou non, émanant des établissements d'enseignement et de recherche français ou étrangers, des laboratoires publics ou privés. 


\title{
Reverse engineering reusable software components from object-oriented APIs
}

\author{
Anas Shatnawi ${ }^{a, b, *}$, Abdelhak-Djamel Seriai ${ }^{a}$, Houari Sahraoui ${ }^{c}, Z^{2}$ akarea Alshara ${ }^{a}$ \\ a UMR CNRS 5506, LIRMM, University of Montpellier, Montpellier, France \\ ${ }^{\mathrm{b}}$ LATECE, University of Quebec at Montreal, Montreal, Canada

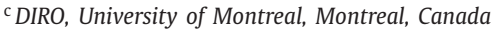

\section{A R T I C L E I N F O}

\section{Article history:}

Received 17 June 2015

Revised 29 February 2016

Accepted 29 June 2016

Available online $\mathrm{xxx}$

\section{Keywords:}

Software reuse

Software component

Object-oriented

API

Reverse engineering

Frequent usage pattern

\begin{abstract}
A B S T R A C T
Object-oriented Application Programing Interfaces (APIs) support software reuse by providing preimplemented functionalities. Due to the huge number of included classes, reusing and understanding large APIs is a complex task. Otherwise, software components are accepted to be more reusable and understandable entities than object-oriented ones. Thus, in this paper, we propose an approach for reengineering object-oriented APIs into component-based ones. We mine components as a group of classes based on the frequency they are used together and their ability to form a quality-centric component. To validate our approach, we experimented on 100 Java applications that used four APIs.
\end{abstract}

(C) 2016 Published by Elsevier Inc.

\section{Introduction}

Nowadays, the development of large and complex software applications is based on reusing pre-existing functionalities instead of developing them from scratch (Frakes and Kang, 2005; Zibran et al., 2011). Application Programming Interfaces (APIs) are recognized as the most commonly used repositories supporting software reuse (Frakes and Kang, 2005). APIs provide a pre-implemented, tested and high quality set of functionalities (Zibran et al., 2011; Monperrus et al., 2012). Consequently, they increase software quality and reduce the effort spent on coding, testing and maintenance activities (Zibran et al., 2011).

In the case of object-oriented APIs, e.g. Standard Template Libraries in $\mathrm{C}++^{+}$or Java SDK, the functionalities are implemented as object-oriented classes. It is well known that reusing and understanding large APIs, such as Java SDK which contains more than 7.000 classes, is not an easy task (Ma et al., 2006; Uddin et al., 2012). On the other hand, classes of an API are used following specific usage patterns, in order to provide services to software applications (Acharya et al., 2007; Wang et al., 2013; Robillard et al., 2013). For example, in the Android API, Activity, GroupView, Con-

\footnotetext{
* Corresponding author.

E-mail addresses: shatnawi@lirmm.fr, anasshatnawi@gmail.com (A. Shatnawi), seriai@lirmm.fr (A.-D. Seriai), sahraoui@iro.umontreal.ca (H. Sahraoui), alshara@lirmm.fr (Z. Alshara).
}

text, LayoutInflater and View are the classes needed to create a simple activity which contains an empty view (Google, 2015). Consequently, many approaches have been proposed, such as Wang et al. (2013); Montandon et al. (2013); Monperrus et al. (2010), to facilitate the understandability and the reusability of APIs by discovering Frequent Usage Patterns (FUPs) of APIs. This is based on the API usage history of software applications (i.e. API clients). Despite the value of FUPs, these are not sufficient to provide a high degree of API reusability and understandability. These are used as guides for reusing API classes and are not themselves reusable entities (Maalej and Robillard, 2013).

Otherwise, software components are admitted to be more reusable and understandable entities than object-oriented ones (Szyperski, 2002). It is because components are considered coarsegrained software entities, while object-oriented classes are considered fine-grained ones. In addition, components define their required and provided interfaces. This means that the component dependencies are more understandable compared to the dependencies among objects. Consequently, many approaches have been proposed to identify components from object-oriented software applications such as Mishra et al. (2009); Kebir et al. (2012); Allier et al. (2011). These approaches aim at mining components by analyzing the source code of software applications. In this context, dependencies between classes are only realized via calls between methods, sharing types, etc. Nevertheless, no approach has been proposed to identify components from object-oriented APIs. In this 
context, we distinguish two kinds of dependencies. The first one is that classes are structurally dependent. The second one is that some classes need to be reused together to implement a functionality. This kind of dependencies cannot be identified by only analyzing the source code, but also needs the analysis of how software applications use the API classes. For example, in the Android API, Activity and Context classes are structurally and behaviorally independent, but they have to be used together to build android applications. This means that classes frequently used together are more favorable to belong to the same component.

In this paper, we propose an approach that aims at recovering software components from object-oriented APIs. This does not only improve the reusability of APIs themselves, but also supports component-based reuse techniques by providing component based APIs. The approach exploits specificity of API entities by statically analyzing the source code of both APIs and their software clients to identify groups of API classes that are able to form components. Our assumption is based on the probability of classes to be reused together by API clients on the one hand, and on the structural dependencies between classes on the other hand. In order to validate the proposed approach, we experimented on a set of 100 Java applications that use three Android APIs in addition to the java.util API. The evaluation shows that structuring object-oriented APIs as component-based ones improves the reusability and the understandability of these APIs.

This journal paper is an extended version of our conference paper published in Shatnawi et al. (2015). The extension includes: (1) A new case study. (2) Deep analysis of the problem (e.g. Section 3). (3) More details and deep analysis of the proposed solution (e.g. how to structure component interfaces). (4) Extended related work analysis. (4) Discussion and threats to validity.

The rest of this paper is organized as follows. Section 2 presents the background needed to understand our approach. Section 3 shows the foundation of our approach. Then, in Section 4 we present the identification of classes composing component interfaces. Section 5 presents how APIs are organized as component-based libraries. Experimentation and results of our approach are discussed through four APIs case studies in Section 6. Next, related works are discussed in Section 7. Finally, concluding remarks and future directions are presented in Section 8.

\section{Background}

\subsection{Component quality model: The ROMANTIC approach}

In this paper, we rely on the component quality model proposed in our previous works related to the ROMANTIC ${ }^{1}$ approach (Kebir et al., 2012; Chardigny et al., 2008a). In ROMANTIC, we have proposed a set of metrics to measure the ability of a group of classes in a software application to form a component. These metrics are defined based on the component quality characteristics that are driven from the component definitions: Composability, Autonomy and Specificity. Composability of a component refers to its ability to be composed through its interfaces without any modification. Autonomy means that a component can be reused in an autonomous way because it encapsulates the strongly dependent functionalities. Specificity refers to the fact that a component implements a limited number of closed functionalities, which makes it a coarse-grained entity.

Similar to the software quality model ISO 9126 (ISO, 2001), we proposed to refine the characteristics of the component into subcharacteristics. Next, the sub-characteristics are refined into the

${ }^{1}$ ROMANTIC: Re-engineering of Object-oriented systeMs by Architecture extractioN and migraTlon to Component based ones. properties of the component (e.g. number of required interfaces). Then, these properties are mapped to the properties of the group of classes from which the component is identified (e.g. group of classes coupling). Lastly, these properties are refined into objectoriented metrics (e.g. coupling metric). This quality refinement model is shown in Fig. 1. According to this model, a quality function has been proposed to measure the component quality. This quality function is used as a similarity metric for a hierarchal clustering algorithm (Kebir et al., 2012; Chardigny et al., 2008a) as well as in search-based algorithms (Chardigny et al., 2008b) to partition the object-oriented classes into groups; where each group represents a component.

\subsection{Frequent usage patterns}

In the domain of data mining, a Frequent Usage Pattern (FUP) is defined as a set of items, subsequences or substructures that are frequently used together by customers (Han et al., 2006). It provides information that helps decision makers (e.g. customer shopping behavior) by mining associations and correlations among a set of items in a huge data set. An example of FUP mining is a market basket analysis. In this example, the customer buying habits are analyzed to identify items that are frequently bought together in the customer shopping baskets, for instance, milk and bread form a FUP when they bought frequently together. The identification of FUP is based on Support quality metric that is used to measure the interestingness of a set of items. Support refers to the probability of finding a set of items in the transactions. For example, the value of 0.30 Support, means that $30 \%$ of all the transactions contain the target item set. The following equation refers to Support:

$S(E 1, E 2)=P(E 1 \cup E 2)$

Where E1, E2 are sets of items; $S$ refers to Support; $P$ refers to the probability.

\section{The proposed approach foundations}

The goal of our approach is at reengineering object-oriented APIs to component-based ones. This is done in two directions. The first one is the identification of groups of classes that can be considered as the object-oriented implementation of the API components. The second one is the identification of how these components can be organized as component-based APIs.

\subsection{Component identification}

We view a component as a group of API classes that provides coarse grained services to clients of an API. Classes that have direct links (e.g. method call, attribute access) with classes implementing other components compose the interfaces of the component. Provided interfaces of a component are defined as a group of methods implemented by classes composing these interfaces. Required interfaces of a component are defined as a group of methods invoked by the component and provided by other components.

The identification of groups of classes composing components is based on two kinds of dependencies; usage-pattern-based and source-code-based ones. Usage-pattern-based is related to the way that software applications use these groups of classes. It refers to observations made based on the analysis of previous usages of APIs. We consider that classes frequently used together are more favorable belonging to a single or a few number of components. This is realized through Frequent Usage Patterns (FUPs) that identify recurring patterns, composed of classes frequently used together. Classes composing FUPs represent the gateways to access the API services. Thus, they are used to guide the identification of classes composing the provided interfaces of components. Classes 
A. Shatnawi et al./The Journal of Systems and Software 000 (2016) 1-19

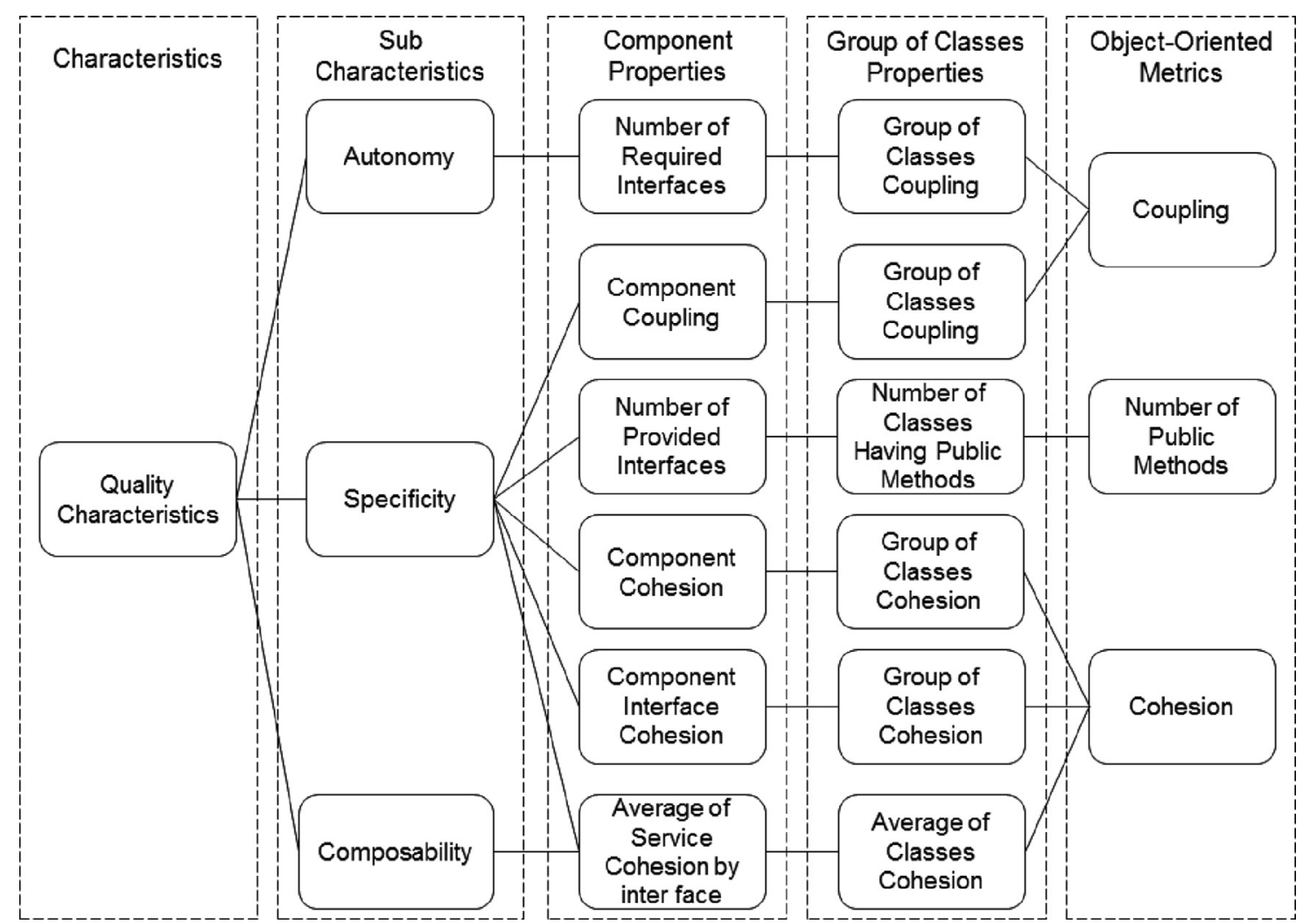

Fig. 1. From component characteristics to object-oriented metrics.

composing a FUP may be related to different services that have been used together. Therefore, they can be mapped to be a part of different component interfaces. Classes of a component interface can be very dependent on other classes that are not directly used by clients of the API. These are identified based on sourcecode-based dependencies. It implies that the component identification process is driven by the identification of its provided interfaces. To this end, the analysis of structural dependencies between classes is used to identify classes forming the core of the component. It is used to form a quality-centric component. This is achieved based on the three quality characteristics that should be satisfied by the group of classes forming the component; Composability, Autonomy and Specificity. To this end, we rely on the component quality model presented by the ROMANTIC approach (Kebir et al., 2012; Chardigny et al., 2008a).

\subsection{API as a library of components}

We organize the API in layers of components. These layers describe how API components are vertically and horizontally organized. We consider that each layer contains components providing services to components of the layer above and requiring services from components of the layer below.

Classes constituting an API can be categorized into two types. The first one is made up of classes that are directly reused by software applications. These represent the implementation of accessible-services of the API (provided to software applications). Thus, components that are identified corresponding to these classes constitute the first layer of the API (i.e. the layer accessed by software applications). The second one is composed of classes representing the rest of API classes. These can also be divided into two categories. The first includes classes providing services to the first layer components. These represent the implementation of components constituting the second layer. In the same manner,

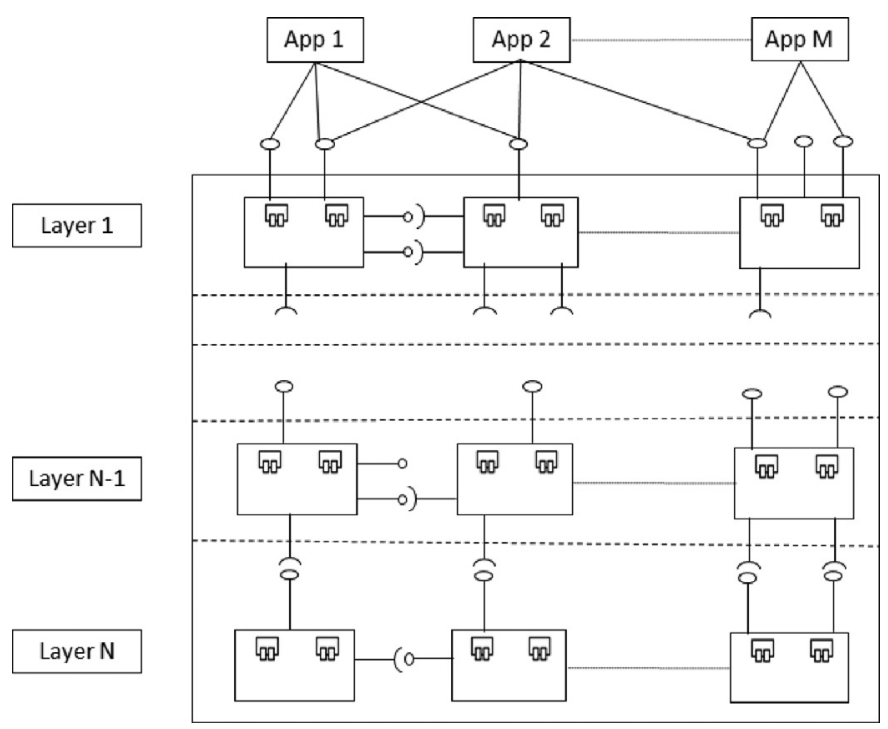

Fig. 2. Multi-layers component-based API.

components composing the other layers are identified. Based on that, we organize component-based APIs as a set of layers describing how their components are organized. Fig. 2 shows our point of view regarding the API organization.

\subsection{Principles and mapping model}

Based on the observations made in the previous sub-sections, the proposed approach can be summarized according to the following principles: 


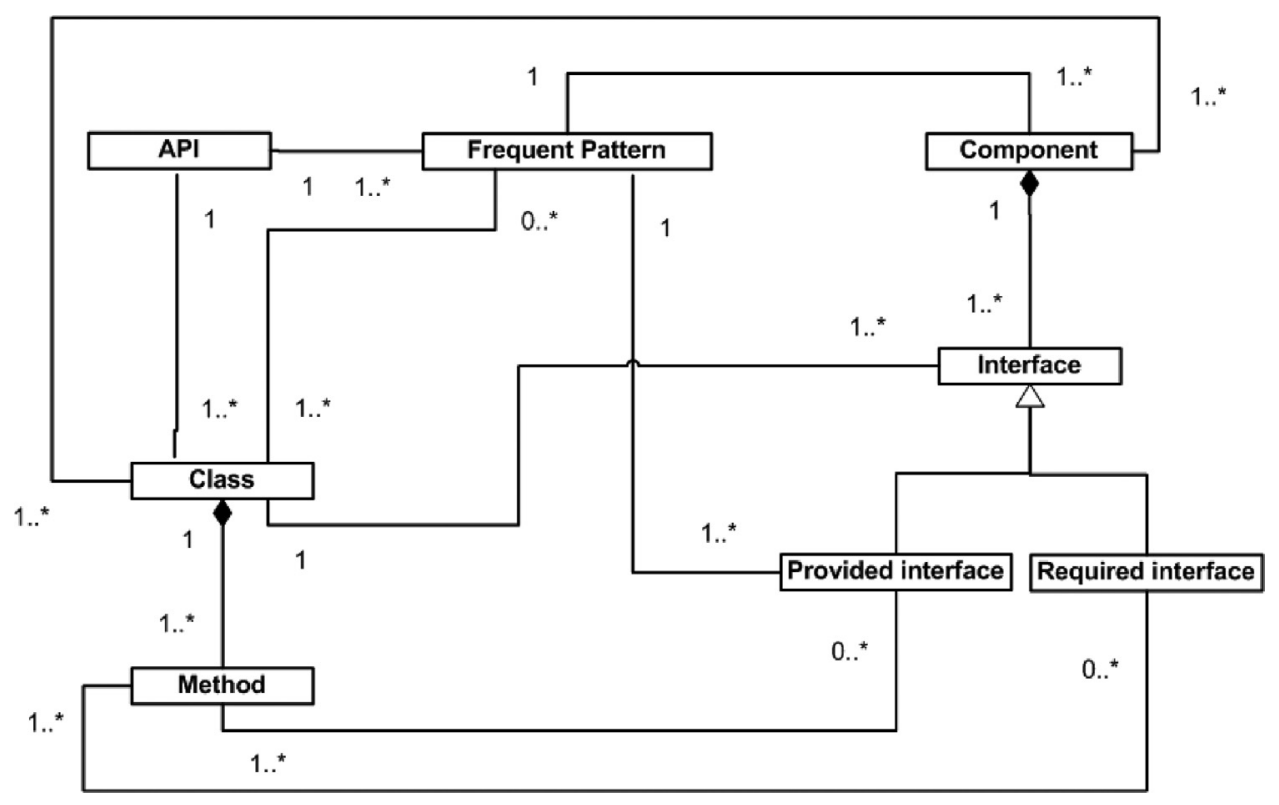

Fig. 3. Mapping class to component through FUP.

- In object-oriented APIs, a component is identified as a group of classes.

- To reengineer the entire object-oriented API into a componentbased one, each class of the API is mapped to be part of at least one component. Each class is mapped either as a class of the component interfaces or as a part of the internal classes of the component.

- Classes frequently used together by software applications provide accessible-user services of the API. Thus, they are used to guide the identification of classes composing the provided interfaces of components. These are identified based on FUPs.

- As a FUP can be composed of classes providing multiple services, its classes can be mapped to be a part of different component interfaces.

- A class of an API can be a part of several FUPs and can participate in implementing multiple services. Consequently, a class can be mapped into multiple component interfaces.

- The identification of classes forming the core of the components is driven by the identification of its provided interfaces.

- The analysis of structural dependencies between classes is used to identify classes forming the core of the component.

- Classes that are not used by software applications are used to structure components of the API layers.

- In a component-based API, the components are vertically and horizontally organized in terms of layers based on the required and provided services between the components.

Based on these principles, we propose a mapping model, shown in Fig. 3, that maps class-to-component through FUPs.

\subsection{Identification process}

We propose the following process to identify components from object-oriented APIs (see Fig. 4):

- Identification of frequent usage patterns. FUPs are identified by analyzing the interactions between the API and its application clients.

- Identification of the interfaces of components. We partition the set of classes of each FUP into subgroups, where each one is considered as related to the provided interfaces of one component (c.f. Fig. 5). The partitioning is based on criteria related to structural dependencies, lexical similarity and the frequency of simultaneous reuse.

- Identification of internal classes of components driven by their provided interfaces. Classes composing the provided interfaces of a component form the starting point for identifying the rest of the component classes. To identify these classes we rely on the analysis of structural dependencies between classes in the API with those forming the interfaces. We check if these classes are able to form a quality-centric component.

- Organizing API as layers of components. As each class of the API must be a part of at least one component, we associate classes that do not compose any of the already identified components to new ones. According to that, we organize component-based APIs as a set of layers. This organization is use-driven. The first layer is composed of components that are used by the software clients, while the second layer is composed of components that provide services used by components of the first layer, and so on. As a result, the API is structured in $N$ layers of components.

\section{Identification of component interfaces}

The identification of classes forming an API component is driven by the identification of classes composing the provided interfaces of this component. Classes composing these interfaces are those directly accessed by the clients of the API. Classes belonging to the same interface are those frequently used together. Therefore, they are identified from frequent usage patterns. Classes of the API composing frequent usage patterns are identified based on the analysis of how API classes were used by the API clients. API classes used together constitute transactions of usage.

\subsection{Extracting transactions of usage}

A transaction of usage is a set of interactions between an API and a client of this API. These interactions consist of calling methods, accessing attributes, inheritance or creating an instance object based on a class of the API. They are identified by statically analyzing the source code of both the API and its clients. Transactions are different depending on the choice of which are the API clients. This choice directly affects the type of the resulting patterns. Mul- 


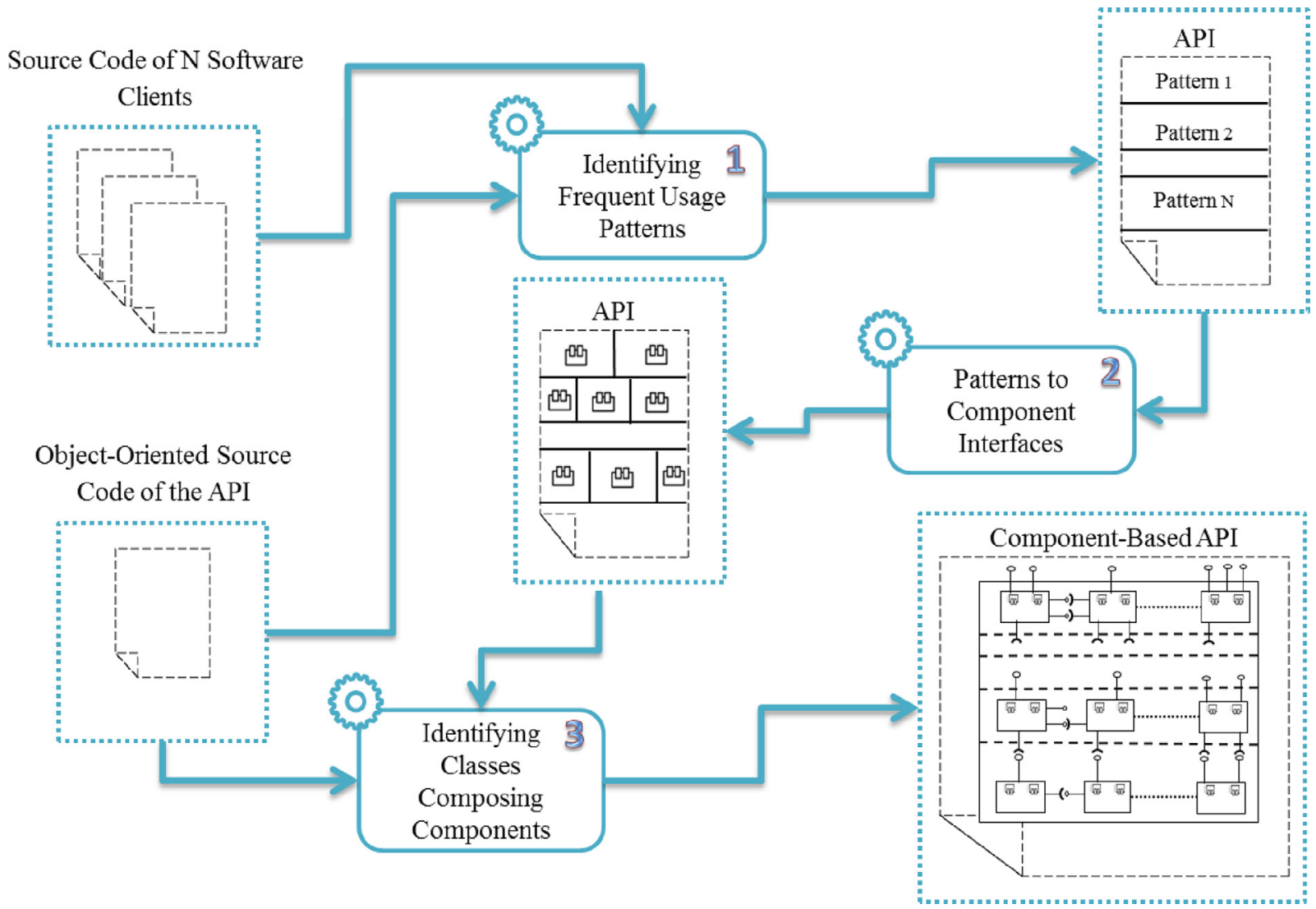

Fig. 4. The process of mining components from an object-oriented API.

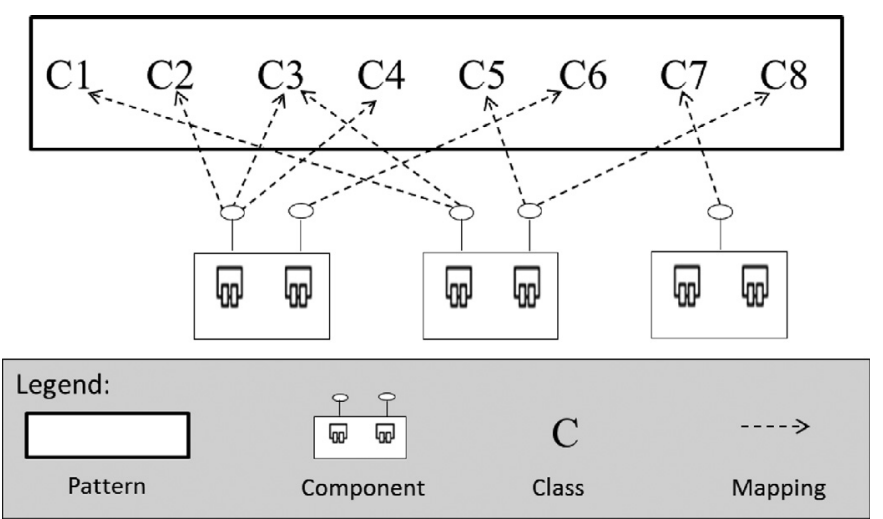

Fig. 5. Identification of provided interfaces of API components from FUPs.

tiple options are possible, a client can be either a class, a group of classes or the whole application. Fig. 6 illustrates these situations. Firstly, if we consider that a transaction corresponding to a class composing a client application, then $\{C 2, C 5\},\{C 3\},\{C 5\},\{C 7\}$ and $\{C 7\}$ are the set of transactions that will be identified based on the first client application. Secondly, if a transaction corresponds to a group of classes from the client application, then $\{C 2, C 5, C 3\}$, $\{C 5, C 7\}$ and $\{C 7\}$ are the set of transactions that will be identified considering the first client application. Thirdly, if a transaction corresponds to the whole client application, then $\{C 2, C 5, C 3, C 7\}$ is the transaction that will be identified considering the first client application.

In our approach, we consider as an API client a group of classes related to the same application functionality. The idea is that classes corresponding to the same application functionality use API classes related to correlated API functionalities. We identify groups of classes related to the same application functionalities as components of this application. This is done thanks to ROMANTIC approach defined in our previous work Kebir et al. (2012). As a result, a transaction is a set of API classes such that each one is used by at least one class of the client component classes. Fig. 7 shows an example. Algorithm 1 shows the process of transaction identifica-

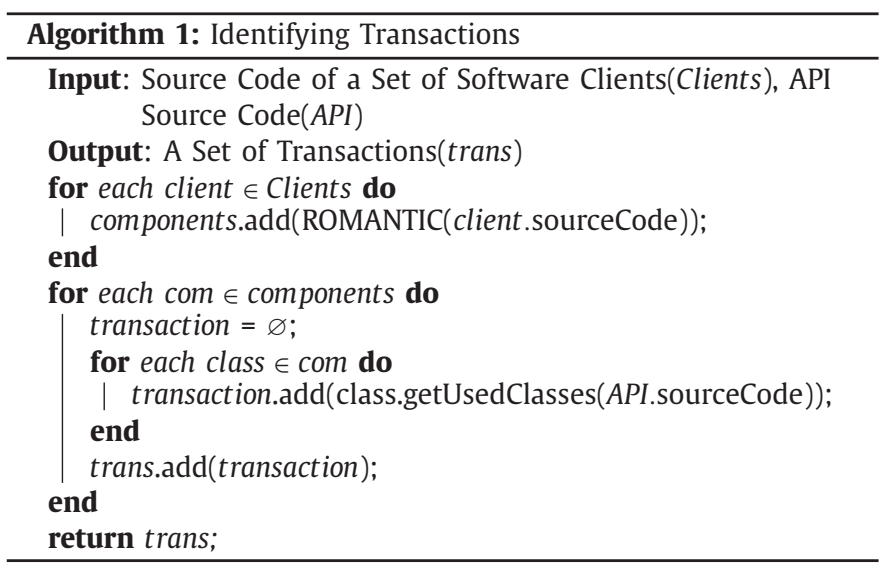

tion. It starts by partitioning each software client into components. Then, for each component, it identifies API classes that are reused by the component classes.

\subsection{Mining frequent usage patterns of classes}

In the previous step, the interactions of application clients with the API are identified as transactions. Based on these transactions, 

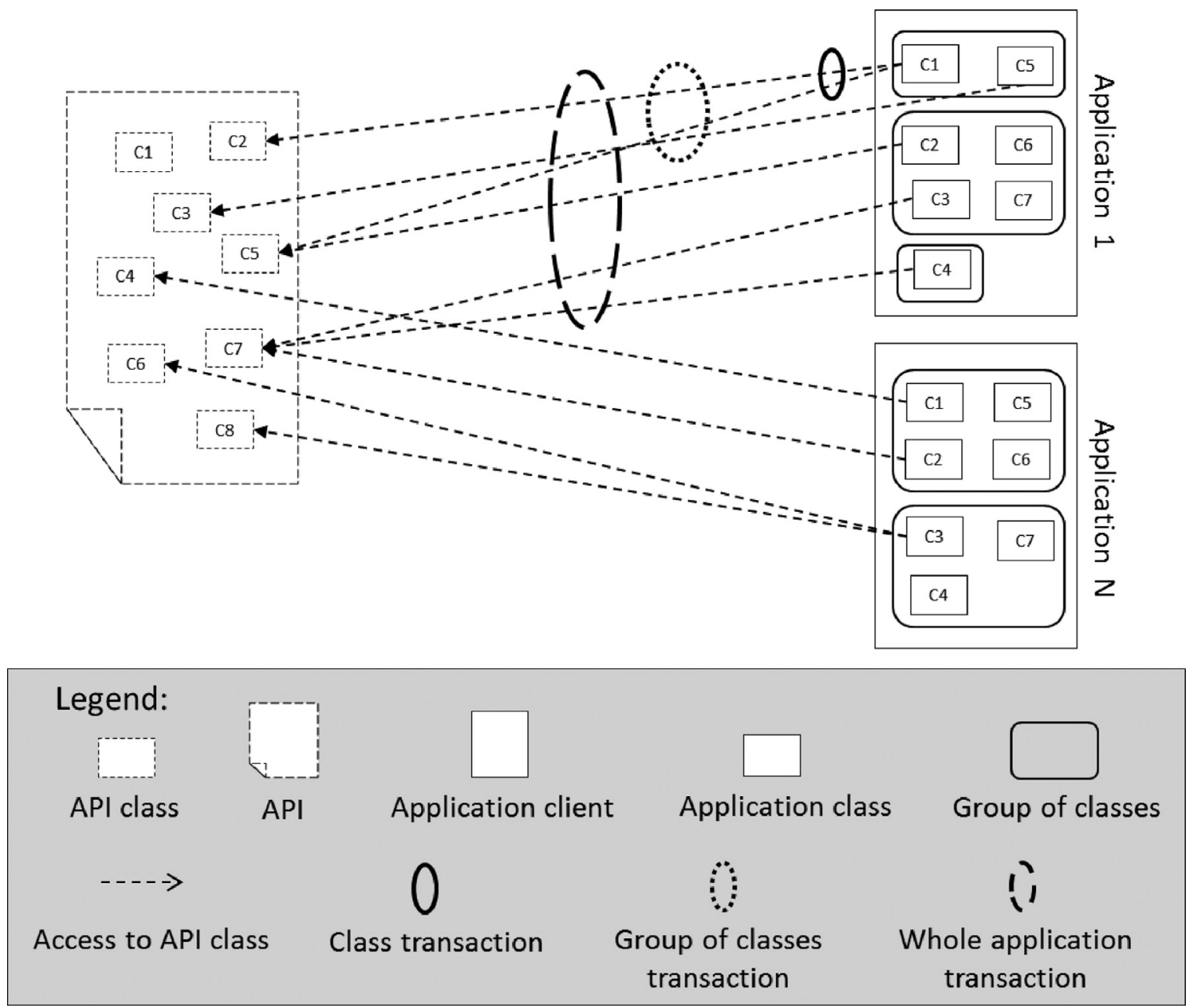

Fig. 6. Transactions based on clients.
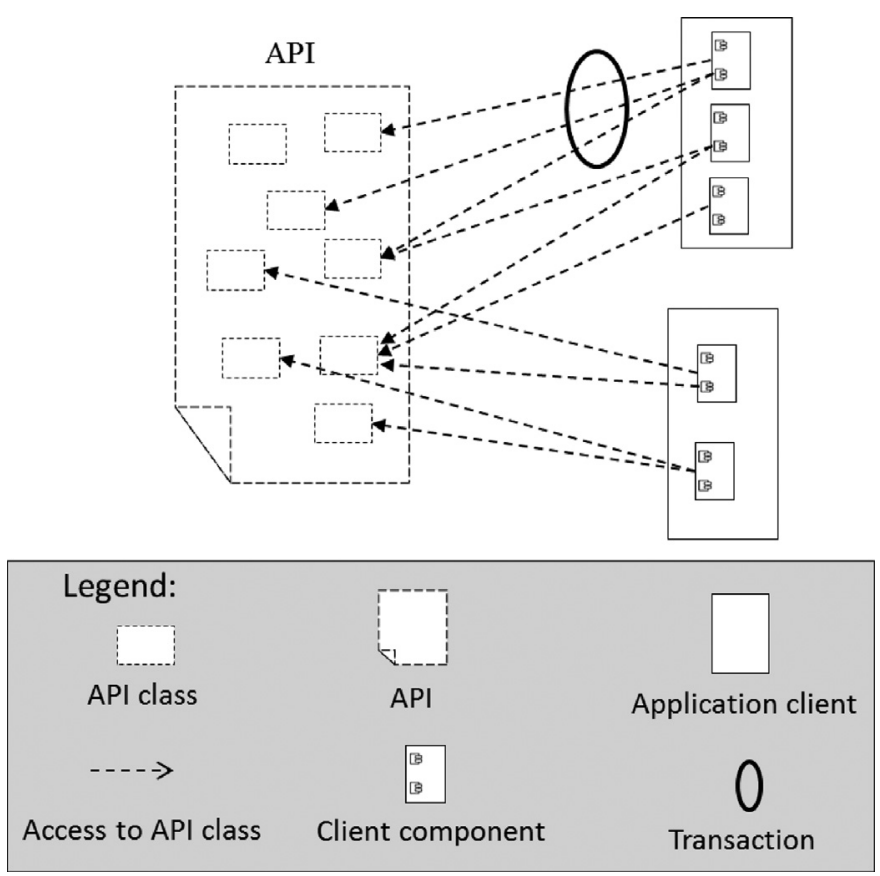

Fig. 7. Client components and corresponding transactions. we identify FUPs. A FUP is defined as a set of API classes that are frequently used together by client components. A group of classes is considered as a frequent pattern if it reaches a predefined threshold of interestingness metric. This metric is known as Support. The Support refers to the probability of finding a set of API classes in the transactions.

\subsubsection{FUPs mining algorithms: an analysis}

The identification of groups of classes forming FUPs can be done based on several algorithms. One of them is the Brute-Force algorithm (Han et al., 2006) that identifies all possible groups of classes. Then, it prunes groups that do not reach the predefined Support threshold value. However, this algorithm is computationally prohibitive since that the identification of all groups, corresponding to $N$ classes, needs $2^{N}$ time complexity (Han et al., 2006). Another algorithm is the apriori algorithm that utilizes the property of anti-monotone (Han et al., 2006), which means that if a group of classes is considered as infrequent, then all of its supersets must be infrequent as well. Thus, they do not need to be generated. However, this algorithm still has to generate the candidate groups of classes. For instance, suppose that we have $10^{4}$ frequent groups of classes of size 1 , it requires to generate about $10^{7}$ groups of size 2. Furthermore, it needs to generate about $10^{30}$ groups of size 10 . Thus, this algorithm does not work in the situation where low Support threshold values are selected (Han et al., 2000). Another algorithm is the Frequent-Pattern Growth (FP Growth) algorithm (Han et al., 2000). In this algorithm, there is no need to produce the candidate groups. Instead, it uses a divide-and-conquer 
Table 1

An example of transactions composed of API classes.

\begin{tabular}{ll}
\hline Transaction ID & List of classes \\
\hline T1 & $\mathrm{C} 1, \mathrm{C} 2, \mathrm{C} 5$ \\
$\mathrm{~T} 2$ & $\mathrm{C} 2, \mathrm{C} 4$ \\
$\mathrm{~T} 3$ & $\mathrm{C} 2, \mathrm{C} 3$ \\
$\mathrm{~T} 4$ & $\mathrm{C} 1, \mathrm{C} 2, \mathrm{C} 4$ \\
$\mathrm{~T} 5$ & $\mathrm{C} 1, \mathrm{C} 3$ \\
$\mathrm{~T} 6$ & $\mathrm{C} 2, \mathrm{C} 3$ \\
$\mathrm{~T} 7$ & $\mathrm{C} 1, \mathrm{C} 3$ \\
$\mathrm{~T} 8$ & $\mathrm{C} 1, \mathrm{C} 2, \mathrm{C} 3, \mathrm{C} 5$ \\
$\mathrm{~T} 9$ & $\mathrm{C} 1, \mathrm{C} 2, \mathrm{C} 3$ \\
\hline
\end{tabular}

\section{Table 2}

Classes ordering inside the transactions.

\begin{tabular}{ll}
\hline Transaction ID & Ordered classes \\
\hline $\mathrm{T} 1$ & $\mathrm{C} 2, \mathrm{C} 1, \mathrm{C} 5$ \\
$\mathrm{~T} 2$ & $\mathrm{C} 2, \mathrm{C} 4$ \\
$\mathrm{~T} 3$ & $\mathrm{C} 2, \mathrm{C} 3$ \\
$\mathrm{~T} 4$ & $\mathrm{C} 2, \mathrm{C} 1, \mathrm{C} 4$ \\
$\mathrm{~T} 5$ & $\mathrm{C} 1, \mathrm{C} 3$ \\
$\mathrm{~T} 6$ & $\mathrm{C} 2, \mathrm{C} 3$ \\
$\mathrm{~T} 7$ & $\mathrm{C} 1, \mathrm{C} 3$ \\
$\mathrm{~T} 8$ & $\mathrm{C} 2, \mathrm{C} 1, \mathrm{C} 3, \mathrm{C} 5$ \\
$\mathrm{~T} 9$ & $\mathrm{C} 2, \mathrm{C} 1, \mathrm{C} 3$ \\
\hline
\end{tabular}

technique to mine FUPs. It firstly builds a special data structure called Frequent-Pattern tree (FP-tree). This tree is used to compress information of class associations. Then, FP Growth divides the FPtree into a collection of databases, such that each one is related to one frequent group of classes.

\subsubsection{Frequent-pattern growth algorithm}

Among the presented algorithms, FP Growth is the best one since that it outperforms the others in terms of time and space complexity (Han et al., 2006). Thus, we mine FUPs based on the FP Growth. To better understand how FP Growth works, we provide an illustrative example. In this example, we have 9 transactions presented in Table 1. The algorithm starts by building the FP-tree corresponding to these transactions. To this end, it first scans the transactions to find the frequency of each API class. In our example, the frequencies of $C 1, C 2, C 3, C 4$ and $C 5$ are respectively 6,7 , 6,2 and 2 . Then, the classes are sorted in a descending order according to their frequency values. That is $C 2, C 1, C 3, C 4, C 5$. Next, the classes inside the transactions are ordered according to their frequency values (see Table 2). Then, the tree is built based on the ordered transactions as follows: starting from the root of the tree, which is labeled by a NULL value, each transaction is added as a branch in the tree, such that the class which has the highest frequency is added first and so on. In the example, the order is $C 2, C 1$, C5 for the first transaction. Whenever a branch shares a common prefix with an already added branch, we only increment the frequency of the shared nodes. Fig. 8 explains the process of building the FP-tree.

Based on the FP-tree, the algorithm extracts conditional pattern bases and a conditional FP-tree for each frequent class. Conditional pattern bases consist of the collection of paths that colocated with the suffix pattern, while the conditional FP-trees are the subtrees that generate the pattern. For example, the conditional pattern bases corresponding to $C 5$ is $\{\{C 2: 1, C 1: 1\},\{C 2: 1, C 1: 1$, C3: 1$\}\}$, thus the conditional FP-tree is $\langle C 2: 2, C 1: 2\rangle$. Paths that do not reach the predefined threshold value are rejected. For example, if the threshold is 2 , the path $\langle C 2: 2, C 1: 2, C 31\rangle$ is excluded since its frequency is 1 . The set of FUPs identified from our example is $\{\{C 2, C 1, C 5\},\{C 2, C 4\},\{C 2, C 1, C 3\},\{C 2, C 1\}\}$.

\subsubsection{Less commonly used classes}

The use of the Support threshold separates the classes of API used by application clients into two groups according to whether they belong to at least one FUP or not. Classes that do not belong to any of the identified FUPs are the less commonly used classes. As each API class that belongs to a transaction is a class that has been accessed by the clients of the API, therefore it must be a part of the classes composing the interfaces of at least one component. We propose assigning each class of the less commonly used classes to the pattern holding the maximum Support value when they are merged together.

\subsection{Identifying classes composing component interfaces from frequent usage patterns}

We identify classes composing component interfaces from those composing FUPs. Each FUP is partitioned into a set of groups, where each group represents a component interface.

\subsubsection{FUP partitioning fitness function}

Classes are grouped together according to three heuristics that measure the probability of a set of classes to be a part of the same interface.

1. Frequency of simultaneous use: classes composing a FUP are regarded differently depending on the frequency of their simultaneous reuse by software applications. As much as classes are reused together, the probability that these classes providing related services is higher. Therefore, we rely on Support metric to measure the association frequency of a set of classes.

2. Cohesion: a group of classes that accesses and shares the same data (e.g. attributes) is probably related to the same service. Thus, we consider that the cohesion of a group of classes is an indication of their functional proximity. To this end, we use LCC metric (Bieman and Kang, 1995) to measure the cohesion of a set of classes. We select LCC since it measures both direct and indirect dependencies between the classes.

3. Lexical similarity: in most cases, classes of an API are welldocumented (i.e. the identifier names are meaningful). Thus, their identifier names indicate to the offered services. Therefore, a group of classes having similar identifier names is likely to belong to the same service. To this end, we utilize Conceptual Coupling metric (Poshyvanyk and Marcus, 2006) to measure classes' lexical similarity based on the semantic information obtained from the source code, encoded in identifiers and comments.

Based on the above heuristics, we propose a fitness function, given below, measuring the ability of a group of classes to form a component interface.

$I Q(E)=\frac{1}{\sum_{i} \lambda_{i}} \cdot\left(\lambda_{1} \cdot \operatorname{LCC}(E)+\lambda_{2} \cdot C C(E)+\lambda_{3} \cdot S(E)\right)$

Where:

- $E$ is a set of object-oriented classes

- $L C C(E)$ is the Cohesion of $E$

- $C C(E)$ is Conceptual Coupling of $E$

- $S(E)$ is the Support of $E$

- $\lambda_{1}, \lambda_{2}$, and $\lambda_{3}$ are weight values, situated in [0-1]. These are used by the API expert to weight each characteristic as needed. 
T1

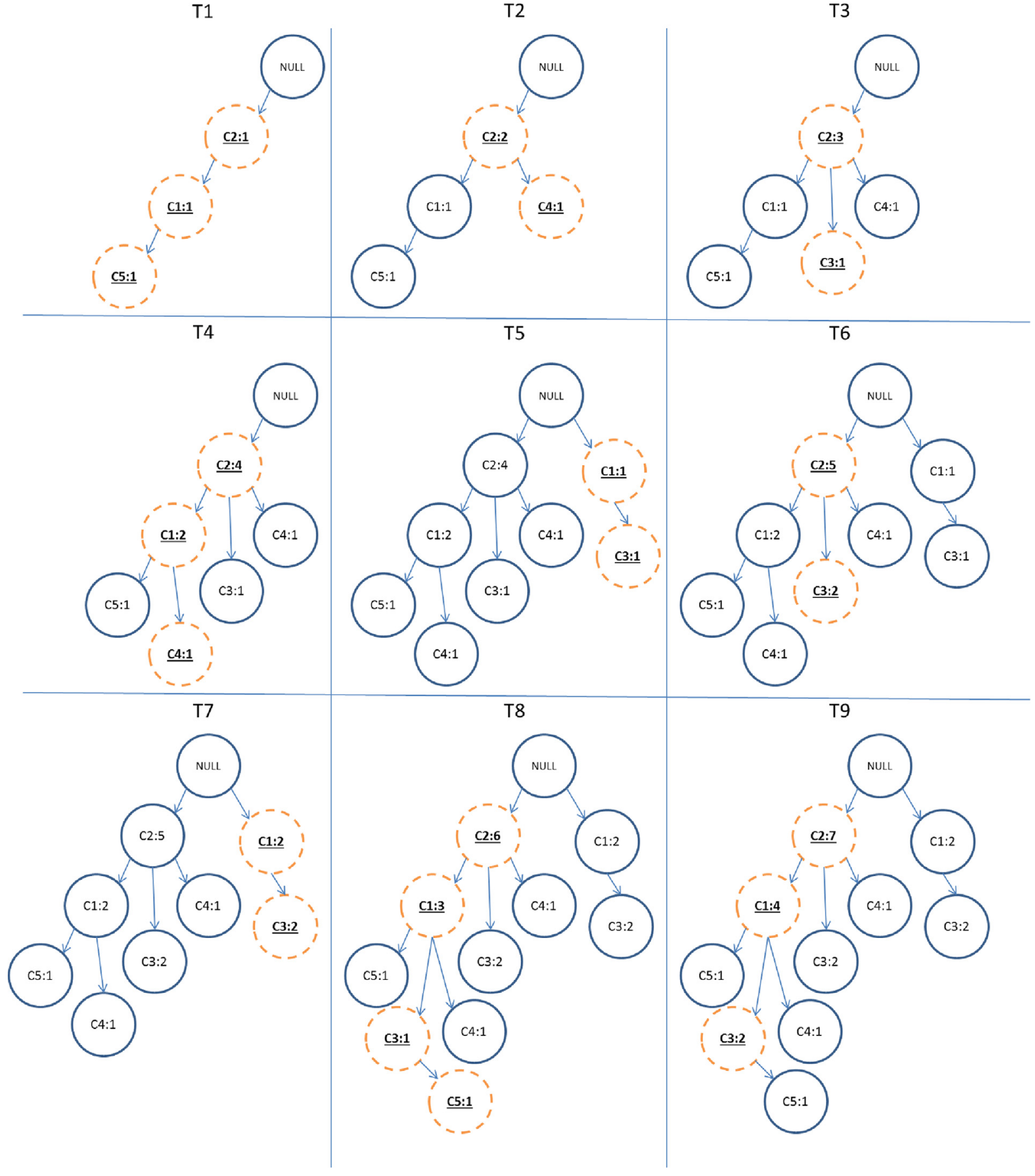

Fig. 8. Process of building the FP-tree.

\subsubsection{FUP partitioning algorithm}

The fitness function defined in the previous section is used to partition each FUP into groups of classes using a hierarchical clustering algorithm. This algorithm consists of two steps. The first one aims to build a binary tree, called dendrogram. This dendrogram provides a set of candidate clusters by presenting a hierarchical representation of classes' similarity. Fig. 9 shows an example of a dendrogram tree, where $C_{i}$ refers to Class $_{i}$. The second step aims at traveling through the built dendrogram, in order to extract the best clusters, representing a partition.

To build a dendrogram, the algorithm starts by considering each individual class as an initial leaf node in a binary tree. Next, the two most similar nodes are grouped into a new one, i.e. as a parent of them. For example, in Fig. 9, the $C_{2}$ and $C_{3}$ classes are grouped. This is continued until all nodes are grouped in the root of the dendrogram. Algorithm 2 presents the procedure used to gather similar classes onto a dendrogram. It takes a set of classes as an input. The result of this algorithm is a hierarchical tree representation of candidate clusters.

To identify the best clusters, a depth first search algorithm is used to travel through the dendrogram tree. It starts from the tree root to find the cut-off points. It compares the similarity of the current node with its children. If the current node has a similarity value exceeding the average similarity value of its children, then 


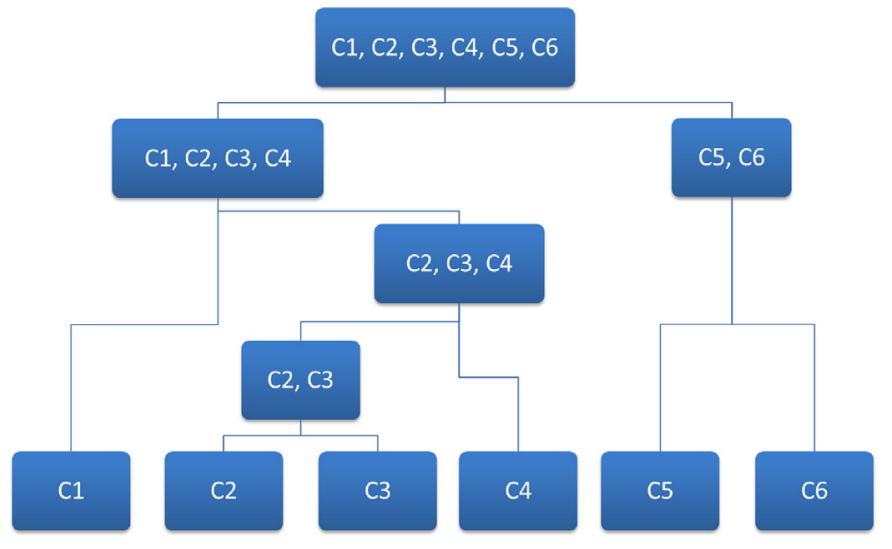

Fig. 9. An example of a dendrogram tree.

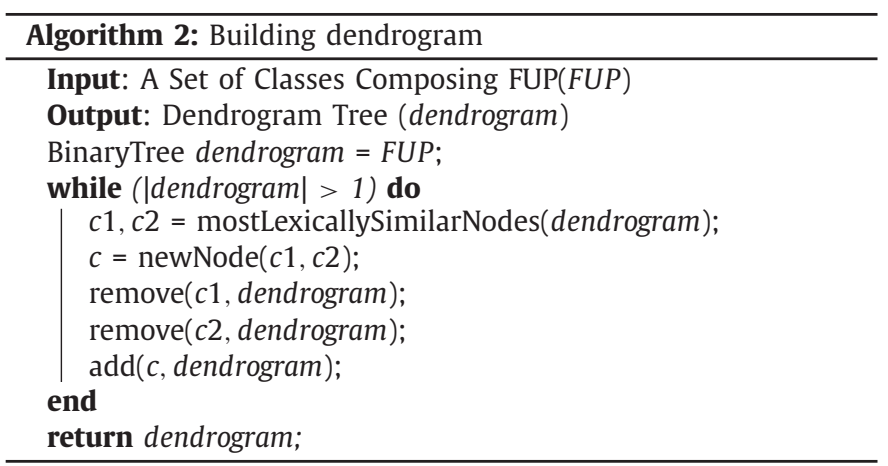

the cut-off point is in the current node where the children minimize the quality function value. Otherwise, the algorithm continues through its children. Algorithm 3 presents the procedure used

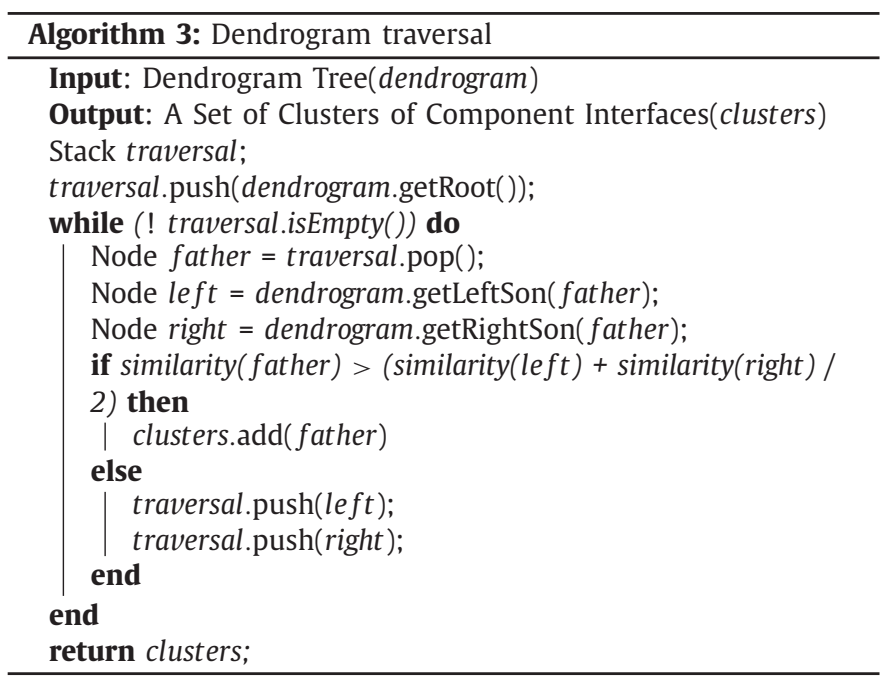

to extract clusters of classes from a dendrogram. The result of this algorithm is a set of clusters, where each contains classes corresponding to a component interface.

\subsection{Structuring component interfaces}

An interface provided by an API component is composed of public methods selected from classes which are identified in the previous step as composing the component interfaces. However, methods of required interfaces of an API component are those called inside one of its classes and defined in classes of other components. These two sets of methods constitute the initial searchspace to identify component interfaces. The identification process is based on the following heuristics to partition this search-space into sub-groups; where each represents an interface:

- Methods that belong to the same interface have a high probability of being used together. Consequently, we consider that methods frequently called together have a higher probability of belonging to the same component interface.

- A group of methods belonging to the same object-oriented interface has a higher probability of belonging to the same component interface.

- A group of methods having a high cohesion and a high lexical similarity has a high probability of belonging to the same component interface.

Based on these heuristics, we define a similarity function that measures the quality of a set of methods to form a component interface. We use Support, LCC (Bieman and Kang, 1995) and Conceptual Coupling (Poshyvanyk and Marcus, 2006) metrics to respectively measure the frequency of use, cohesion and lexical similarity of a set of methods. This function is defined as follows:

$$
\begin{aligned}
\operatorname{Interface}(M)= & \frac{1}{\sum_{i} \lambda_{i}} \cdot\left(\lambda_{1} \cdot \operatorname{LCC}(M)+\lambda_{2} \cdot \operatorname{CS}(M)\right. \\
& \left.+\lambda_{3} \cdot S(M)+\lambda_{4} \cdot \operatorname{SOI}(M)\right)
\end{aligned}
$$

Where:

- $M$ is a set of methods.

- $L C C(M), C S(M), S(M)$, and $S O I(M)$ respectively refers to the cohesion, Cosine similarity, support and the association with the same object-oriented interface $(1$ if yes, else 0$)$ of $M$.

- $\lambda_{1}, \lambda_{2}, \lambda_{3}$ and $\lambda_{4}$ are weight values, situated in [0-1]. These are used by the API expert to weigh each characteristic as needed.

Based on this similarity function, we partition methods of the above search-space into clusters based on a hierarchical clustering algorithm. Each cluster contains a set of methods forming an interface.

\section{API as library of components}

\subsection{Identifying classes composing components}

As we mentioned before, the component identification process is driven by the identification of its provided interfaces. This means that API classes forming a component are identified in relation to their structural dependencies with the classes forming provided interfaces of the component. Thus, classes having either direct or indirect links with the interface ones compose the search-space of classes that may be added to the component. The selection of a group of classes, from the search-space, is based on the measurement of the quality of the component, when they are included.

To identify the best group of classes that can serve as the implementation of a component providing the identified interfaces, we investigate all subsets of candidate classes. Then, the set that maximizes the component quality is selected. However, this requires an exponential time complexity to identify all subsets (i.e. NP-hard problem). Thus, we present a heuristic-based technique that identifies near-optimal groups of classes of the corresponding optimal ones.

The identification of these classes is done gradually. In other words, we start to form the group of classes composing the interface ones, and then we add other classes to form a component based on the component quality measurement model. Classes having either direct or indirect links with the interface ones represent the candidate classes to be added to the component. At each 


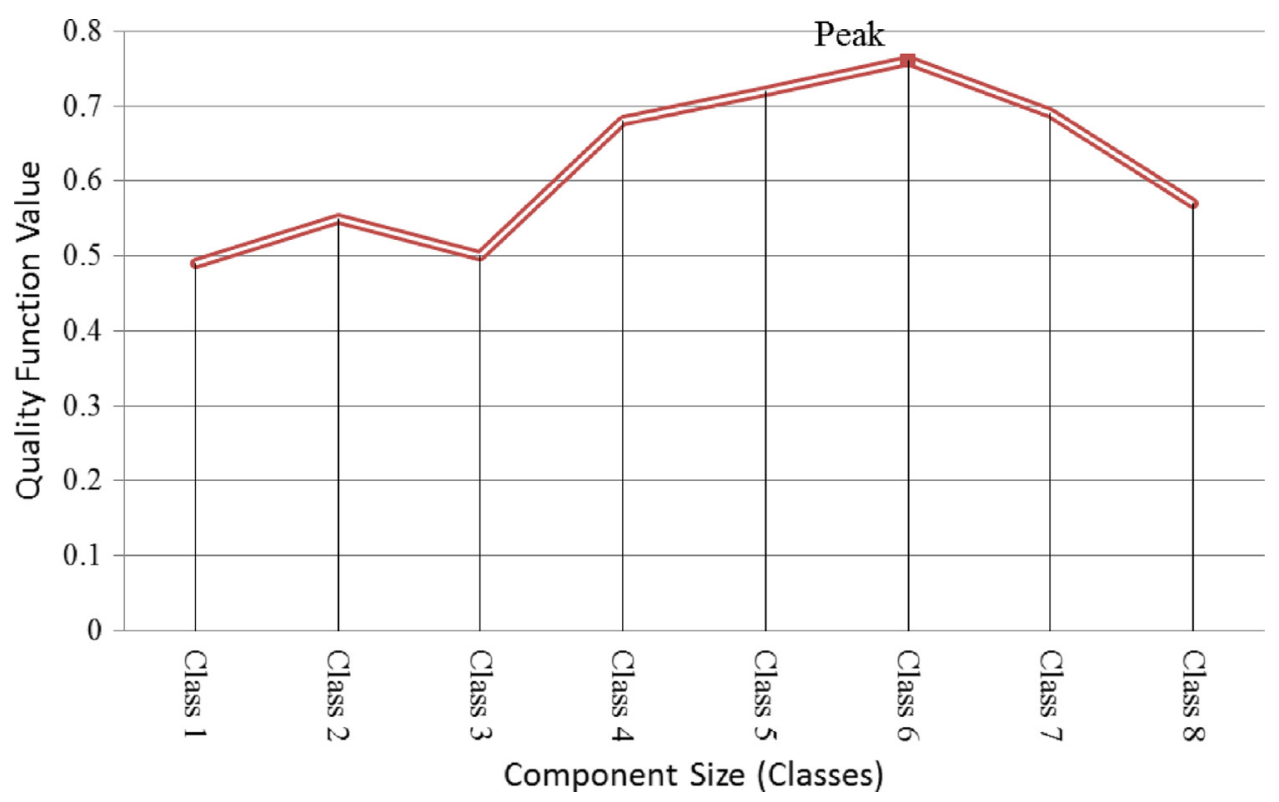

Fig. 10. Identifying classes composing components.

step, we add a new API class. This is selected based on the quality value of the component, formed by adding this class to the ones already selected. The class that maximizes the quality value is selected in this step. This is done until all search-space classes are investigated.

Each time we add a class, we evaluate the component quality. Then, we select the peak quality value to decide which classes form the component. This means that we exclude classes added after the peak value. As an example, Class 7 and Class 8 in Fig. 10 are excluded from the resulting component because they were added after the quality value reached the peak. Algorithm 4 illustrates

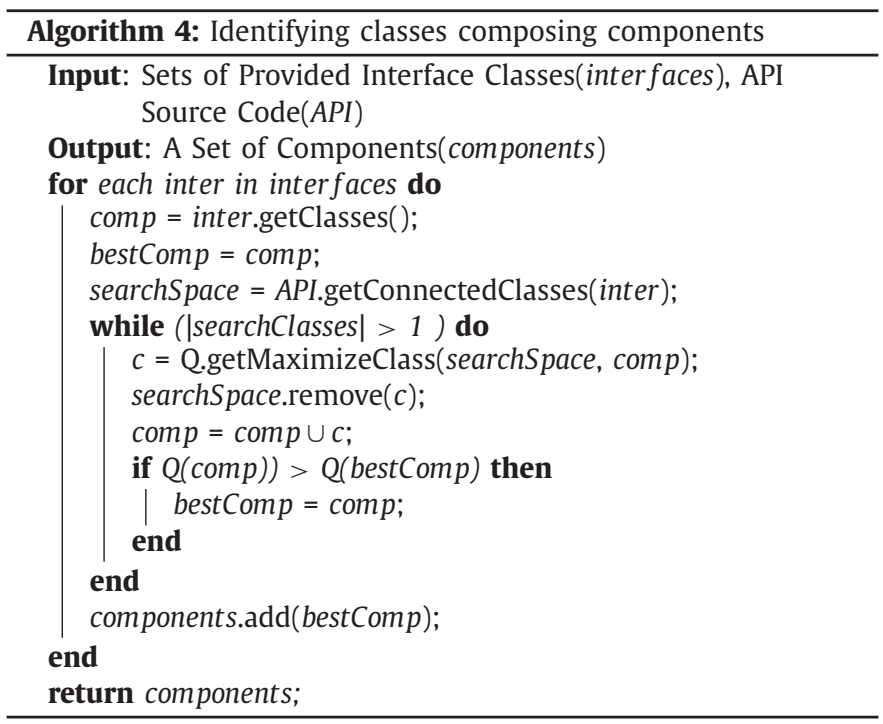

the process of identifying classes composing a component. In this algorithm, Q refers to the quality fitness function.

\subsection{Organizing API as layers of components}

As we previously mentioned, the API is structured in $N$ layers of components. To identify components of layer $L$, we rely on components of layer $L-1$. We proceed similarly to the identifica- tion of the components of the first layer. We use required interfaces of the components already identified in layer $L-1$ to identify the interfaces provided by components in layer $L$. This continues until reaching a layer where its components either do not require any interface or they require ones already identified. Fig. 11 shows an example that illustrates how the components composing each layer are identified, where Fig. 11a presents an object oriented API, Fig. 11b shows how the first layer components are identified, Fig. 11c explains the second layer component identification and Fig. 11d shows the resulting component-based API.

Each interface that is defined as required for a component of layer $L-1$ is considered as provided by a component of layer $L$ except ones provided by the already identified components. The identification of these interfaces is similar to the identification of provided interfaces of the first layer. Thus, we consider that each component (already identified) in layer $L-1$ is a client of the rest of API classes. This means that we collect a set of transactions, such that each transaction consists of classes that are used by a component in layer $L-1$. These transactions are used to identify FUPs based on the FP Growth algorithm. Similar to the first layer, each FUP is divided into groups of classes composing provided interfaces of components in layer $L$. The partitioning is based on (i) the cohesion of classes, (ii) the lexical similarity of these classes and (iii) the frequency of their simultaneous use. Analogously to the identification of the components of the first layer, the other classes composing each component are identified starting from classes composed of its already identified provided interfaces. Algorithm 5 shows the procedure that identifies component-based API as a set of layers composed of components.

\section{Experimentation and results}

\subsection{Experimental design}

\subsubsection{Data collection}

We collected a set of 100 Android-Java applications from open-source repositories. ${ }^{2}$ These applications are from different domains, such as communication, education, puzzle and so. This

\footnotetext{
2 sourceforge.net, code.google.com, github.com, gitorious.org, and aopensource. com
} 


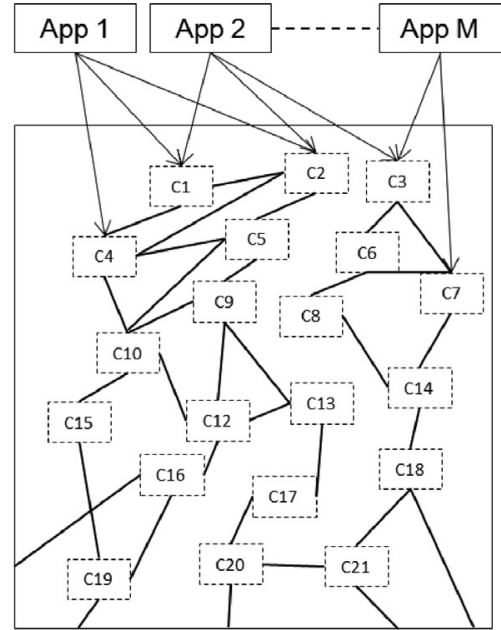

(a)

Object-oriented API

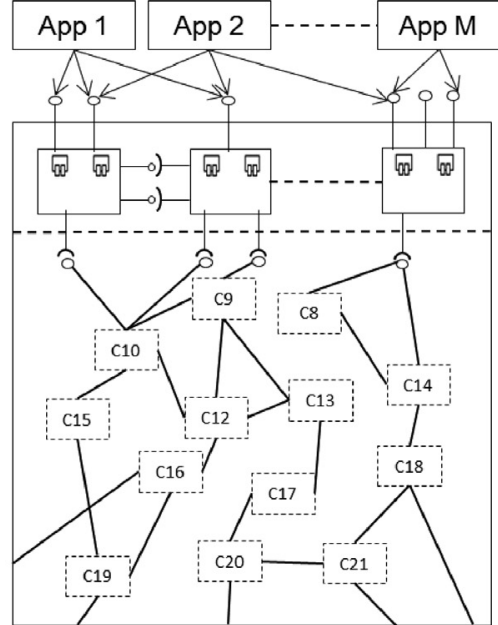

(b)

$1^{\text {st }}$ layer components

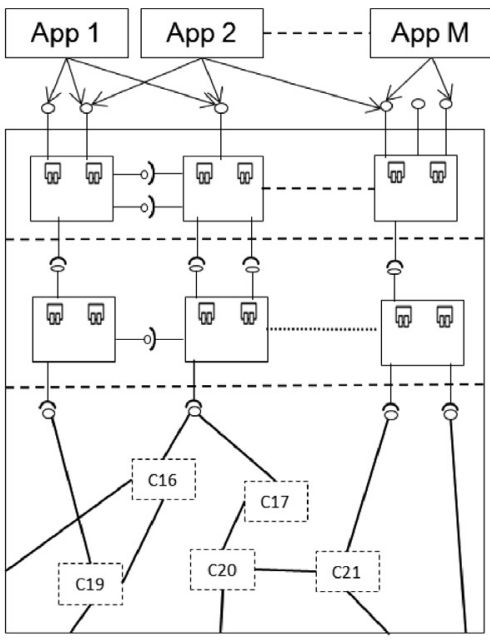

(c) $2^{\text {nd }}$ layer components

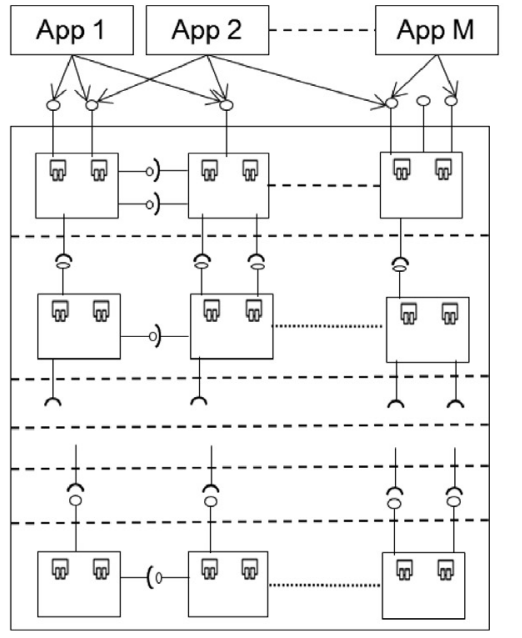

(d)

$\mathrm{N}$ layer components

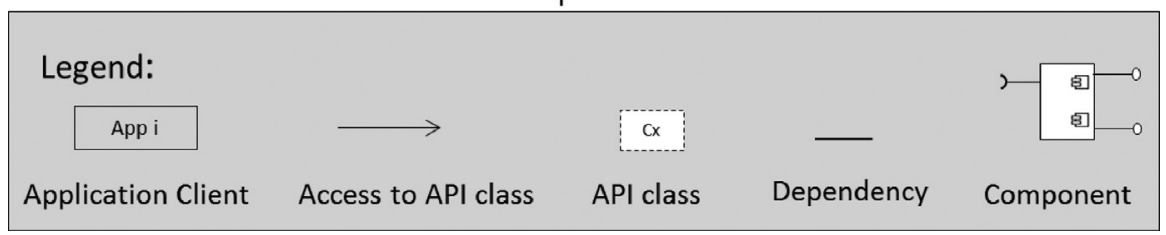

Fig. 11. Identifying component-based API as layers of components.

allows to test the approach on clients that are very different from each other. The average size of these applications in terms of number of classes is 90 . Table 3 presents the categorization of the applications. We distinguish 19 categories based on Google Play labeling. The applications are developed based on classes of the Android $S D K K^{3}$ In our experimentation, we focus on four APIs. The first one is the android.view API composed of 491 classes. This API provides services related to the definition and management of the

\footnotetext{
${ }^{3}$ We select android API level 14 as a reference.
}

user interfaces in android applications. The second API is the android.app API composed of 361 classes. This API provides services related to creating and managing android applications. The third one is the java.util API composed of 846 classes. The fourth API is the android API that is composed of 5790 classes. which includes all of the android services.

\subsubsection{Research questions and evaluation method}

The approach is evaluated on the collected software applications and APIs. We identify client components independently for each software application. Each component in software is consid- 


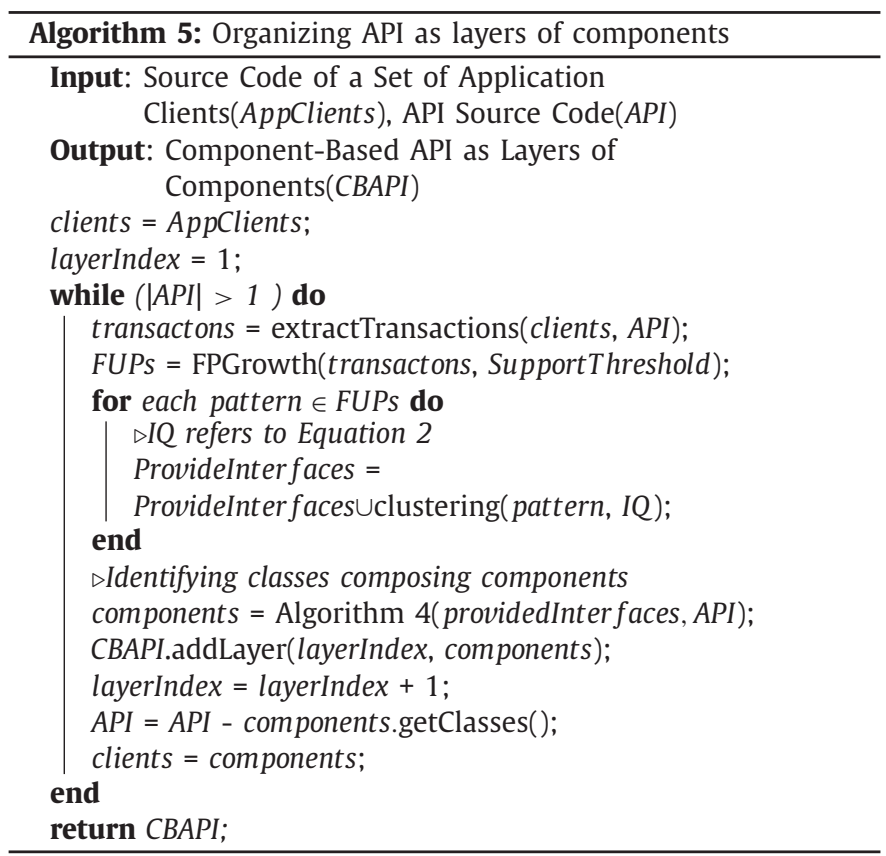

ered as a client of the APIs to form a transaction of classes. Then, we mine Frequent Usage Patterns (FUPs) from the identified transactions. Next, from classes composing each FUP, we identify classes composing a set of component interfaces. Then, we identify all component classes starting from ones composing their interfaces. Lastly, the results related to component-based APIs obtained based on our approach are presented.

We evaluate the obtained components by answering the three following research questions.

- RQ1: Do the Resulting Component-Based APIs Reduce the Understandability Efforts? This research question aims at measuring the saved efforts in the API understandability that are brought by migrating object-oriented APIs into componentbased ones.

- RQ2: Are the Mined Components Reusable? As our approach aims at mining reusable components, we evaluate the reusability of the resulting component. This is based on measuring how much related classes are grouped into the same components.

- RQ3: Is the Identification of Provided Interfaces Based on FUPs Useful? The proposed approach identifies the provided interfaces of the components based on how clients have used the API classes (i.e. FUPs). Thus, this research question evaluates how much benefit the use of FUPs brings by comparing components identified by our approach with the ones identified without taking FUPs into account.

\subsection{Results}

\subsubsection{Intermediate results and identified components}

The average number of client components identified from each software is 4.5 and the average number of classes composing each component is 18.73 . Table 4 shows the average number of transactions per software application (ANTIC), the average transaction size in terms of classes (ATS), and the percentage of components that have used the API $(P C U)$. The last column of this table shows an example of transactions.

The results show that android, view, app and java.util APIs have been used respectively by only $54 \%, 29 \%, 32 \%$ and $49 \%$ of client components. In addition, we note that each client component has used the API classes intensively compared to the number of classes composing it. For example, the transaction size is 17.91 classes for the view API, where the average number of classes per component is 18.73. This is due to the fact that classes that serve the same service in software applications, and consequently depend on the same API classes, are grouped together in the same client component.

The identification of FUPs relies on the value of the Support threshold. The number and the size of the mined FUPs depend on this value. For all application domains where FUPs are used (e.g. data mining), this value is determined by domain experts. In our approach, to help API experts to determine this value, we assign

Table 3

The categorization of the selected android applications.

\begin{tabular}{|c|c|c|}
\hline Category & Application client names & Sum \\
\hline Personalisation & ADW Launcher & 1 \\
\hline Tool & $\begin{array}{l}\text { Alerts, Alogcat, AppsOrganizer, CH-EtherDroid, CVox, CalendarPicker, CidrCalculator, ColorPicker, Countdown, } \\
\text { CountdownTimer, DiskUsage, FileManager, Gcstar, Hermit, Introspy, LegoMindstroms, Look, MotionDetection, Prey, } \\
\text { PubkeyGenerator, PwdHash, SuperGenPass }\end{array}$ & 22 \\
\hline Communication & $\begin{array}{l}\text { AndroidomaticKeyer, AutoAnswer, ConnectBot, CorporateAddressBook, Dialer2, ExchangeOWA, GetARobotVPNFrontend, } \\
\text { LibVoyager, PhotSpot, Swiftp }\end{array}$ & 10 \\
\hline Education & ARviewer, Mandelbrot & 2 \\
\hline Productivity & AVP, CamTimer, DroidStack, OpenIntents, QueueMan & 5 \\
\hline Arcade & AndorsTrail, Dolphin, GITron, MAME4droid, AlienbloodBath, VectorPinball & 6 \\
\hline Puzzle & $\begin{array}{l}\text { AndroMaze, ASquare, Blokish, CrossWord, Dazzle, Holoken, Lexic, Mathdoku, NewspaperPuzzles, OpenSudoku, } \\
\text { WordSearch }\end{array}$ & 11 \\
\hline $\begin{array}{l}\text { Local, Travel and } \\
\text { Transport }\end{array}$ & $\begin{array}{l}\text { AripucaTracker, Avare, BigPlanetTracks, BostonBusMap, CustomMaps, DriSMo, GoHome, GoogleMapsSupport, } \\
\text { OnMyWay, OpenMap, RateBeerMobile }\end{array}$ & 11 \\
\hline Augmented Reality & AugmentRealityFW, DroidAR & 2 \\
\hline Media & BansheeRemote, BiSMoClient, LiveMusic, ChanImageBrowser, FloatingImage, MediaPlayer & 6 \\
\hline Health and Fitness & BinauralBeats, CompareMyDinner, DIYgenomics, Pedometer & 4 \\
\hline News & DroidLife, FeedGoal, Phoenix & 3 \\
\hline Action & Doom & 1 \\
\hline Weather & AussieWeatherRadar & 1 \\
\hline Photography & AsciiCam & 1 \\
\hline Shopping & ARMarker & 1 \\
\hline Social & Historify, LookSocial, Tumblife & 1 \\
\hline Card & HotDeath & 3 \\
\hline Finance & Ministocks & 1 \\
\hline Others & DistLibrary, GeekList, HeartSong, LocaleBridge, Macnos, NGNStack, SwallowCatcher, GraphView & 8 \\
\hline
\end{tabular}


Table 4

The identification of transactions.

\begin{tabular}{lllll}
\hline API & ANTIC & ATS & PCU & Example \\
\hline android & 2.61 & 64.82 & 0.54 & Bitmap, Path, Log, Activity, Location, Canvas, Paint, ViewGroup, MotionEvent, View, TextView, GestureDetector \\
view & 1.51 & 17.91 & 0.29 & Menultem, Menu, View, ContextMenu, WindowManager, MenuInflater, Display, LayoutInflater \\
app & 1.58 & 10.90 & 0.32 & ProgressDialog, Dialog, AlertDialog, Activity, ActionBar, Builder, ListActivity \\
java.util & 2.34 & 30.21 & 0.49 & Queue $<$ Character $>$, Matcher, Calendar, Collection $<$ Character $>$, Pattern, Locale, Arrays, List, TimeZone \\
\hline
\end{tabular}

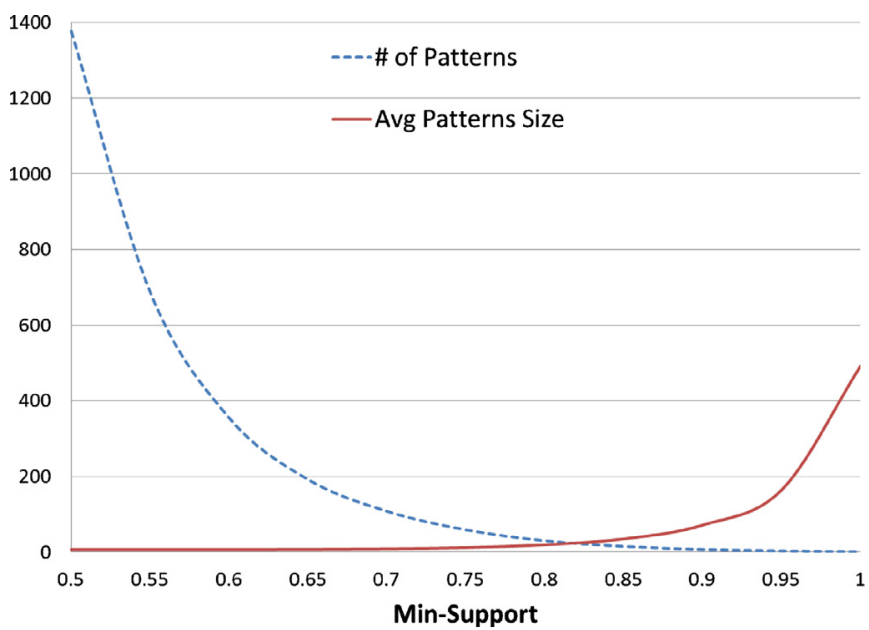

Fig. 12. Changing the support threshold value to mine FUPs in android API.

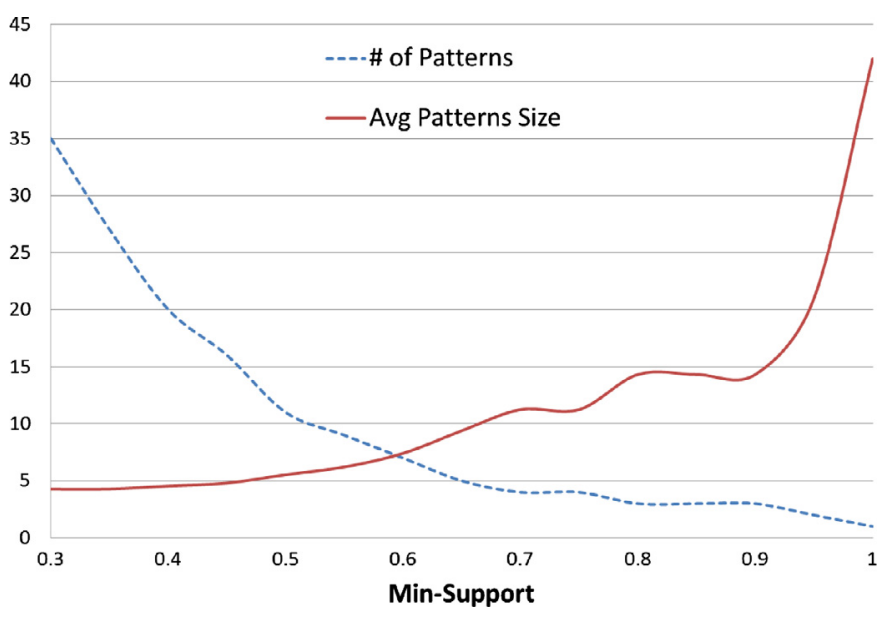

Fig. 13. Changing the support threshold value to mine FUPs in view API.

the Support threshold values situated in [0\%-100\%]. We give for each Support value the number of the mined FUPs and the average size of the mined FUPs for each API. Figs. 12-15 respectively refer to the results of the android, the view, the app and the java.util APIs. The results show that the number of mined FUPs is directly proportional to the Support value, while the average size of the mined FUPs is inversely proportional. We note that the java.util API has a different behavior compared to the other APIs in terms of the relationship between the Support values and the number of FUPs corresponding to the expected functionalities. As it is shown in Fig. 15, the accepted number of FUPs can be reached at low Support values situated in [0.05-0.30\%]. These low Support values refer to the fact that the java.util has diverse functionalities that are used by client applications in a deserve way, thus it requires low Support values to separate them into different FUPs.

Based on their knowledge of the API, API experts can select the value of the Support. For example, if the known average number

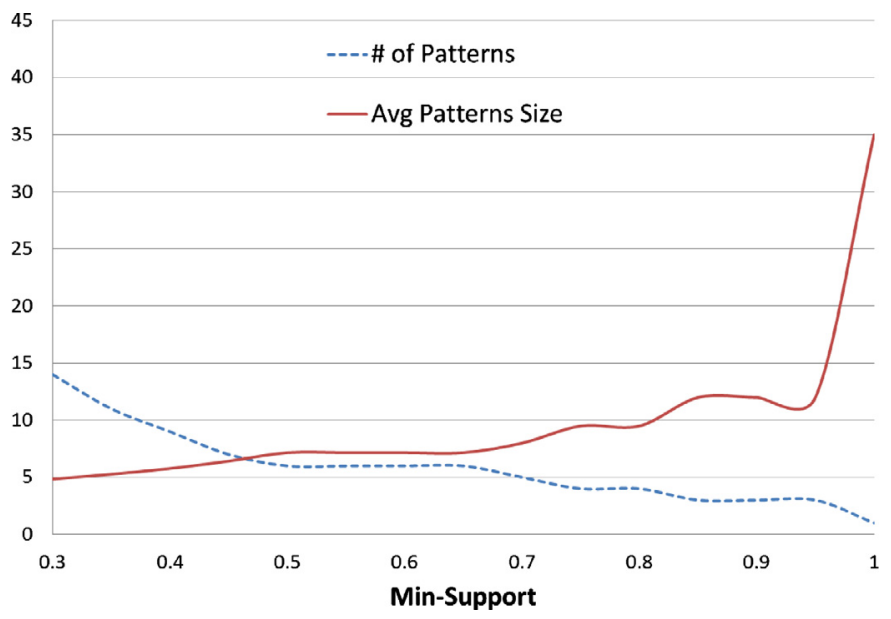

Fig. 14. Changing the support threshold value to mine FUPs in app API.

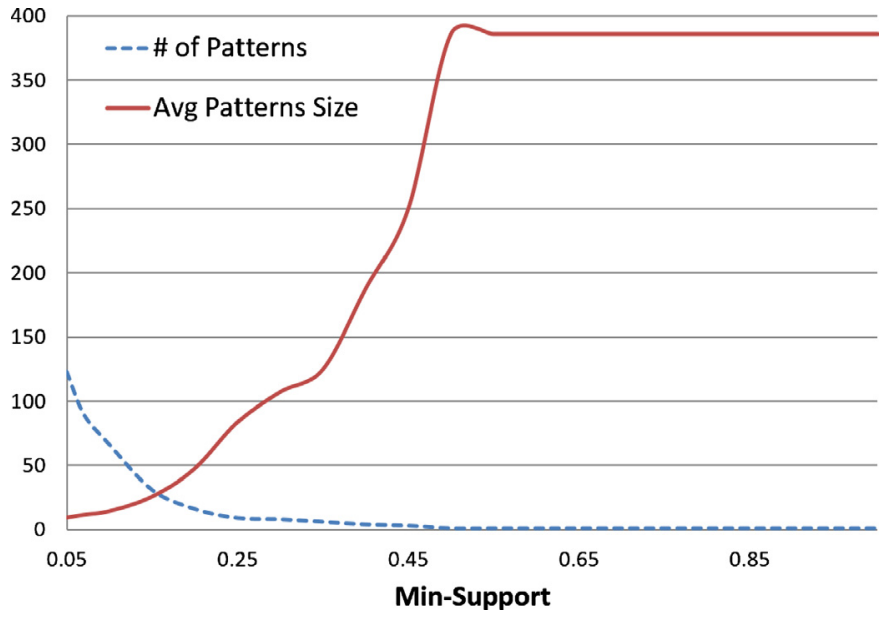

Fig. 15. Changing the support threshold value to mine FUPs in java.util API.

of API classes used together to implement an application service is $N$, then the experts can choose the Support value corresponding to FUPs having $N$ as the average size. Based on the obtained results and our knowledge of android APIs, ${ }^{4}$ we select the Support threshold values as $60 \%, 45 \%, 45 \%$ and $15 \%$ respectively for the android, the view, the app and the java.util APIs.

Table 5 shows examples of the mined FUPs. For instance, the FUP related to view API contains 10 classes. The analysis of this FUP shows that it corresponds to three services: animation (Animation and AnimationUtils classes), view (Surface, SurfaceView, SurfaceHolder, MeasureSpec, ViewManager and MenuInflater classes), and persistence of the view states (AbsSavedState and AccessibilityRecord classes). These services are dependent. Animation service needs the view service and the data of animation view needs to be persistent.

\footnotetext{
4 The authors of this paper are experts on the android APIs
} 
Table 5

Examples of the mined FUPs.

\begin{tabular}{ll}
\hline API & Example \\
\hline $\begin{array}{l}\text { android } \\
\text { view }\end{array}$ & Intent, Context, Log, SharedPreferences, View, TextView, Toast, Activity, Resources \\
app & Surface, Animation, AnimationUtils, AccessibilityRecord, ViewManager, MenuInflater, AbsSavedState, SurfaceView, SurfaceHolder, MeasureSpec \\
java.util & Dialog, Activity, ProgressDialog \\
\hline
\end{tabular}

Table 6

Identification of component interfaces from FUPs.

\begin{tabular}{lllll}
\hline API & ANCIP & ACIS & TNCI & Examples \\
\hline android & 1.57 & 5.62 & 232 & Activity, View, TextView, Toast \\
view & 2.17 & 2.94 & 19 & Surface, SurfaceView, SurfaceHolder \\
app & 2.50 & 4 & 10 & Dialog, ProgressDialog \\
java.util & 3.84 & 2.78 & 46 & Calendar, HashMap, Date, List, Locale, Timer \\
\hline
\end{tabular}

Table 7

Identifying classes composing components.

\begin{tabular}{llrl}
\hline API & NMC & \multicolumn{1}{c}{ ACS } & Example \\
\hline android & 232 & 19.99 & Activity, View, TextView, Toast, Drawable, GroupView, Window, Context, ColorStateList, LayoutInflater \\
view & 19 & 7.49 & Surface,SurfaceView, SurfaceHolder, MockView, Display, CallBack \\
app & 10 & 5.86 & Dialog, ProgressDialog, AlertDialog \\
java.util & 85 & 9.73 & Calendar, HashMap, Date, List, Locale, Timer, TimerHeap, TimerTask, AbstractMap, Map,TimeZone, SimpleTimeZone \\
\hline
\end{tabular}

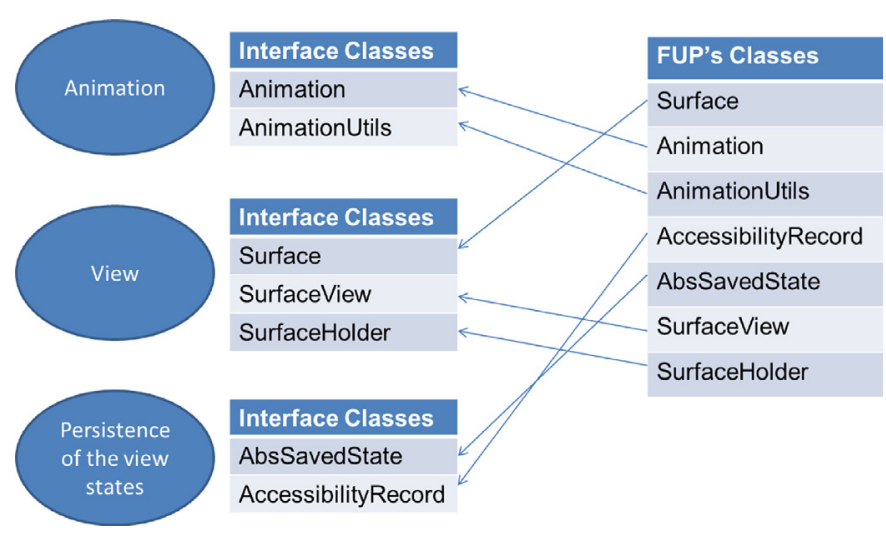

Fig. 16. An instance of partitioning a FUP into component interfaces from view API.

In Table 6, we present the results of interface identification in terms of the average number of component interfaces identified from a FUP $(A N C I P)$, the average number of classes composing component interfaces (ACIS) and the total number of component interfaces in the API (TNCI). The last column of this table presents examples of component interfaces identified from the FUPs given in Table 5.

The results show that FUPs contain classes corresponding to a different set of services. On average, each FUP is divided into $1.57,2.17$ and 2.5 services, such that each service is provided by 5.62, 2.94 and 4 classes respectively for android, view and app APIs. Fig. 16 shows an instance of partitioning a FUP into component interfaces from view API. The analysis of classes composing the identified component interfaces shows that they are related to three services; animation, view and persistent of the view states.

Table 7 presents the results related to the mined components composing the first API layer. For each API, we give the number of the mined components $(N M C)$ and the average number of classes composing the mined components $(A C S)$. The last column of this table shows examples of classes composing components initially identified from classes composing provided component interfaces presented in Table 6 . The results show that the services offered
Table 8

The final results.

\begin{tabular}{llll}
\hline API name & API entity & API size & No. of used entities \\
\hline \multirow{2}{*}{ android } & OO & 5790 & 491 \\
& $C B$ & 497 & 54 \\
view & OO & 491 & 42 \\
\multirow{4}{*}{ app } & $C B$ & 43 & 17 \\
\multirow{3}{*}{ java.util } & OO & 361 & 45 \\
& OO & 55 & 5 \\
& $C B$ & 846 & 468 \\
\hline
\end{tabular}

by classes of android, view and app APIs are identified as 232, 19 and 10 components respectively. This means that developers only require to interact with these components to get the required services from these APIs.

Table 8 shows the final results obtained from our approach. For each API, we firstly give the size of the API in terms of the number of object-oriented classes composing the API and the number of the identified components. Secondly, we present the total number of used entities (classes and respectively components) by the software clients. The results show that classes involved in providing related services are grouped into one component. Furthermore, the total number of cohesive and decoupled services is identified for each API. For instance, android API consists of 497 components (coarse-grained services), while view, app and java.util APIs contain 43, 55 and 85 components respectively.

\subsubsection{Answering research questions}

RQ1: Do the Resulting Component-Based APIs Reduce the Understandability Efforts? The efforts spent to understand an API is directly proportional to the complexity of the API. This complexity is related to the number of API elements and the individual element's complexity. On the one hand, the reduction in the number of elements composing the API is obtained by grouping classes collaborating to provide one coarse-grained service into one component. The results show that the average number of identified components for the studied APIs is $11 \%(((497 / 5790)+(43 / 491)$ $+(55 / 361)) / 3$ ) of the number of classes composing the APIs. This 


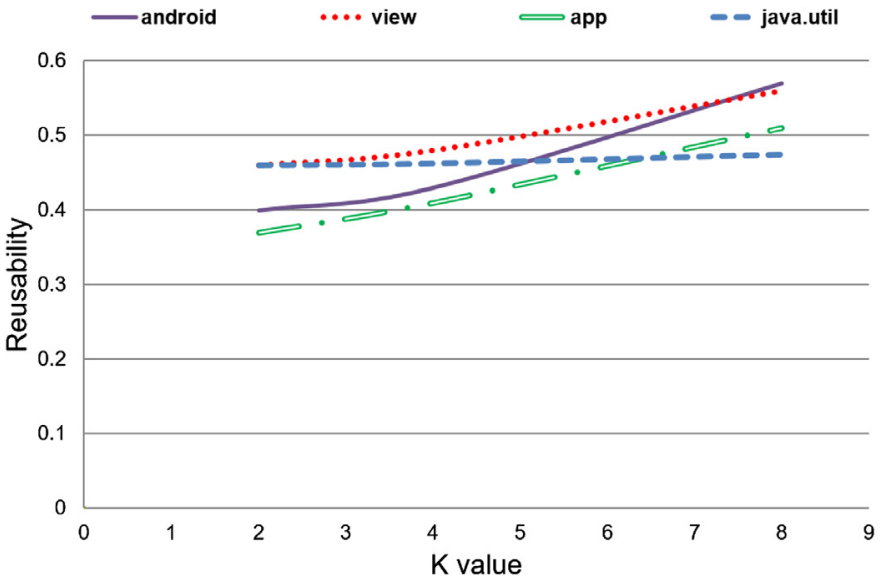

Fig. 17. Reusability validation results.

means that the API size is significantly reduced by mapping classto-component. On the other hand, the reduction in the individual element complexity is done by migrating object-oriented APIs into component-based ones. Meaning, components define their required and provided interfaces, while object-oriented classes at least do not define required interfaces (e.g. a class may call a large number of methods belonging to a set of classes without an explicit specification of these dependencies). The results show that the average number of used components for the APIs is $4 \%$ $(((54 / 491)+(17 / 42)+(5 / 45)) / 3)$ of the number of used classes. This means that the effort spent to understand API entities is significantly reduced in the case of software applications developed based on API components compared to the development based on API classes. Note that, developers only need to understand the component interfaces, but not the whole component implementation.

RQ2: Are the Mined Components Reusable? We consider that the reusability of a software component is related to the number of used classes among all ones composing the software component. Thus, we calculate the reusability of the component based on the ratio between the numbers of used classes composing the component to the total number of classes composing the component. To prove that our resulting component-based APIs could be generalized to another independent set of client applications, we rely on $K$-fold cross validation method (Han et al., 2006). The main idea is to evaluate the model using independent client applications. Thus, K-fold divides the set of client applications into $K$ parts. Then, the identification process is applied $K$ times by considering, each time, $K-1$ different parts for the identification process and by using the remaining part to measure the reusability. Next, we take the average of all $K$ trial results. In our experiment, we set $K$ to 2,4 , and 8 .

Fig. 17 presents the results of this measurement. These results show that the reusability results are distributed in a disparate manner. The reason behind this dispersion is the size of the train and test data as well as the size of the API. For instance, the average reusability for the app API is $37 \%$ when the number of train clients is 50 application clients, while it is $51 \%$ when the number of train clients is 88 application clients. The reusability of java.util is slightly enhanced when the number of its client applications is increased. We interpret this by the fact that java.util is an extensive API that contains utility functionalities used in similar patterns by the all client applications. Thus including more client applications only adds little enhancement of the reusability. Still, a larger number of application clients increase the reusability of the resulting components.

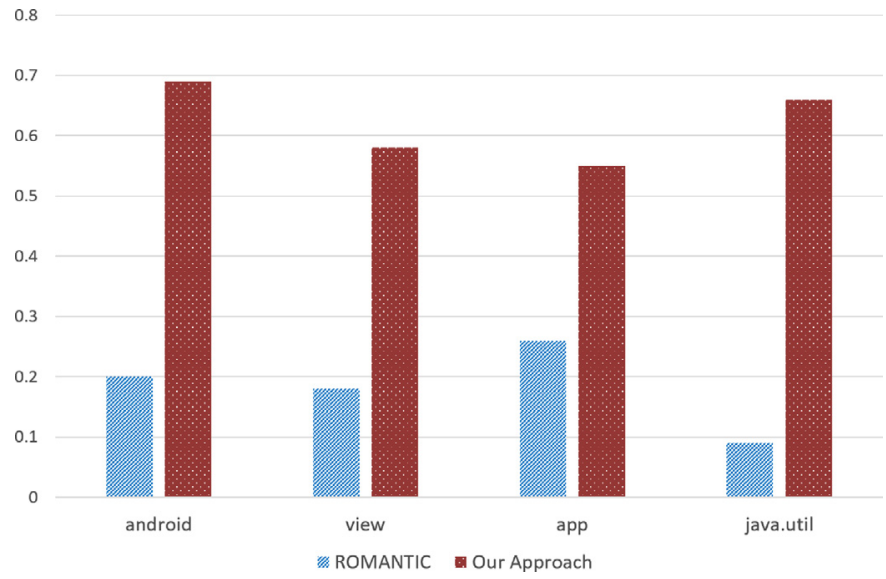

Fig. 18. Density validation results.

RQ3: Is the Identification of Provided Interfaces Based on FUPS Useful? To prove the utility of using FUPs during the identification process, we compare the components mined based on our approach with ones mined using the ROMANTIC approach, which does not take FUPs into consideration. This is based on the density of use of the component provided interfaces by application clients. The density refers to the ratio between the number of used interface classes to the total number of interface classes for each component. Fig. 18 shows the average density for the two identification approaches. These results show that our approach outperforms the ROMANTIC approach. For instance, the application clients need to reuse a larger number of components mined based on the ROMANTIC with less density of provided interface classes compared to components mined based on our approach. For instance, the average usage density of classes composing provided interfaces of ROMANTIC components is $18 \%$, while it is $62 \%$ for components mined by our approach for all APIs. Also, the results show a significant difference between the density values obtained by respectively applying ROMANTIC and our approach. This is due to the fact that classes of java.util API are not very coupled and cohesive. These relationships (coupling and cohesion) are used by ROMANTIC to group classes together to identify components. In contrast, our approach allows to group these classes together because they are frequently used together.

\subsection{Threats to validity}

Our proposed approach is subjected to two types of threats: internal validity and external validity.

\subsubsection{Threats to internal validity}

There are five aspects to be considered regarding the internal validity. These are as follows:

1. The validations of understandability and reusability of the resulted component-based APIs are not directly measured. On the one hand, the understandability is measured through the complexity of the resulted API, while in some cases a complex API can be understandable if it is well documented. However, for the same API, the understandability of a complex version is worse than the understandability of a less complex one, even if both versions are already documented. On the other hand, the reusability is measured based on the number of used classes among the ones composing the components. Although the reusability of components needs to be measured based on their interfaces, this provides an indication of how the component interfaces will be reused by the future software clients. 
2. We use FPGrowth algorithm to mine FUPs. Nevertheless, this algorithm has a limitation of ignoring those classes whose patterns' support values do not reach the support threshold (i.e. less commonly used classes). Thus, some of the API classes may not be presented by a FUP. However, we attach each class of them to a FUP holding the maximum support value when it is added. This guarantees that each API class used by software applications is attached to at least one FUP.

3. As our approach is use-driven, the results depend on the quality and the number of usages of the API. This means that identified FUPs rely on the considered software clients. Therefore, the identification of provided interfaces and their corresponding components depends on API clients. Consequently, it is essential to select clients having the largest number of usages of the API.

4. In the case of facing a NP-hard problem, we rely on heuristic algorithms instead of optimal algorithms. This affects the accuracy of the results. However, these heuristics guarantee near-optimal solutions, such as clustering algorithms.

5. There are two polymorphism categories; method overloading and method overriding (Benlarbi and Melo, 1999). In our analysis, we used a static technique that ables to detect the method overloading polymorphism, but not the method overriding polymorphism. The letter can be only detected at the runtime (dynamic analysis). The major problem of dynamic analysis is the difficulty to identify a sufficient number of real use scenarios to cover the maximum of possible execution paths.

\subsubsection{Threats to external validity}

There are two aspects to be considered regarding the external validity. These are as follows:

1. We experiment on APIs, as well as client applications, that are implemented using Java programming language. The obtained results can be generalized for other object-oriented languages due to the fact that FUPs mining is not influenced by existing variability in the object-oriented programing languages (e.g. multi inheritance). Only, the measurement of the coupling and the cohesion can be influenced by these variants. This requires an adapted algorithm for measuring the coupling and the cohesion based on the additional object-oriented dependencies (e.g. friend class in $\mathrm{C}++$ ).

2. The way that API classes are reused together may strongly depend on the choice of the client applications, i.e. different client applications may use API classes following different patterns, ending up in different components. This may impact the reusability of the identified components for new independent applications. However, our assumption is that software developers follow very similar reuse patterns even for different applications belonging to different domains. Robillard et al. provide (Robillard et al., 2013) a survey of approaches that successfully utilized this assumption to identify API documentation (Uddin et al., 2012), recommendation systems (Zhang et al., 2012), improving bug detection (Monperrus et al., 2010), etc. To evaluate this assumption in our context, we selected 100 client applications that are related to different domains. Based on Google Play, they are about 19 domains (see Table 3). This coupled with the usage of K-fold validation method indicate that the resulting component-based APIs is reusable for new independent clients even they belong to different domains. In addition, the results show that the reusability is increased as well as the number of input clients increases. Therefore, we recommend to select as much as possible of API client applications to minimize the influence of domain specific API usages.

\section{Related work}

To the best of our knowledge, no approach has been proposed to identify components from object-oriented APIs. However, we present three research areas that are related to our approach. The first one aims at identifying components by analyzing objectoriented software applications. The second area aims to identify features from software applications. The third one is related to mining frequent patterns of API usage.

\subsection{Identifying software components from software applications}

APIs and software applications are different compared to relationships between classes composing them. In the case of objectoriented applications, classes composing them are structural and behavioral dependent to provide the expected services. For instance, these dependencies are realized via calls between methods, sharing types, etc. For APIs, we distinguish two kinds of dependencies. On the one hand, classes are structural and behavioral dependent, to provide reusable services for software applications. On the other hand, some classes needs to be reused together, i.e. simultaneously, by software applications to implement API services (e.g. JFrame and Layout classes in java.swing API). This kind of dependencies can not be identified by only analyzing the API source code, but also needs the analysis of how software applications use the API classes.

Dependencies between classes composing object-oriented applications are exploited by numerous approaches that aim to identify components from object-oriented applications (Garcia et al., 2013; Ducasse and Pollet, 2009). In von Detten et al. (2013), Detten et al. presented the Archimetrix approach, which aims at mining the architecture of legacy software. It relies on a clustering algorithm to partition the system classes into components. This algorithm depends on name resemblance, coupling and cohesion metrics as a fitness function. In Kebir et al. (2012), Kebir et al. presented an approach to extract components from a single objectoriented software system. Classes composing the extracted components form a partition. Mined components are considered as a part of the component-based architecture of the corresponding software. In Allier et al. (2011) Allier et al. depended on dynamic dependencies between classes to recover components. Based on the use case diagram, the execution trace scenarios are identified. Classes that frequently occur in the execution traces are grouped into a single component. Cohesion and coupling metrics are also taken into account during the identification process. Weinreich et al. proposed, in Weinreich et al. (2012), an approach to recover multi-view architecture models of software applications implemented based on service oriented architecture. The authors classified software artifacts based on the information from source code, configuration files and binary codes. In Erdemir et al. (2011), the author extracted the architecture of an object-oriented software using the fast community detection algorithm. Also, a performance evaluation of fast community and five clustering algorithms is applied. The authors converted the object oriented elements into a graph representation. Then, the algorithms are applied to identify the most connected component within the graph. Lastly, software architects analyze and evaluate the resulted architecture. In Shatnawi and Seriai (2013), an approach has been presented to mine reusable components from a set of similar software applications. A component is considered as more reusable, when it is reused many times by the software applications. The authors firstly identified components independently from each software application. Then, based on the lexical similarity between the classes composing these components, they identified reusable ones. In Duszynski et al. (2011), an approach was presented to visually analyze the distribution of variability and commonality 
among the source code of product variants. The analysis includes multi-level of abstractions (e.g. line of code, method, class, etc.). This aims to facilitate the interpretation of variability distribution, to support identifying reusable entities.

\subsection{Feature mining from software applications}

The difference between feature mining and component identification arises from the difference between a feature and a component. The difference is also in the goals and nature of the process. A feature is a non-structural element that provides "uservisible aspect, quality, or characteristic of a software system or systems" (Kang et al., 1990). It does not have any interfaces that represent the interaction between features, rather than component interfaces, required and provided ones. In addition to that, features and components belong to different levels of abstraction, where software requirements are abstracted at a high level as features, while a component represents an architectural element at the design level.

There are many approaches presented to address feature location and feature identification. These aims to identify program units such as methods, or classes that represent features. In Dit et al. (2013), a survey of them is presented. These approaches identify features based on the analysis of single software application, such as Antoniol and Guéhéneuc (2005); Chen and Rajlich (2000); Damaševičius et al. (2012), and multiple software applications, such as Xue (2011); Ziadi et al. (2012).

\subsection{Mining frequent patterns of API usage}

FUPs are observations made based on the analysis of previous uses of APIs. They aim to help users of APIs by identifying recurring patterns, composed of API elements frequently used together. FUPs and components serve reuse needs in two different ways. Components are entities that can be directly reused and integrated into software applications, while FUPs are guides for reuse and not entities for reuse. In addition, components and FUPs are structurally different. Classes composing a component serve a coherent body of services, while a FUP may be related to different services. Dependencies of component's classes are mostly internal, which forms an autonomous entity. FUP's can be very dependent on other API classes that are not directly used by clients of APIs. A component is structured and reused via interfaces, while FUPs are not directly reusable entities.

Several approaches have been proposed to mine FUPs based on the analysis of API clients. Robillard et al. provide a survey of these approaches (Robillard et al., 2013). These approaches can be mainly classified based on four main criteria. The first one is related to the goal, which can be either giving examples and recommendations of how to use API entities such as (Montandon et al., 2013; Uddin et al., 2012), supporting the documentation of APIs like (Montandon et al., 2013; Wang et al., 2013), or improving the bug detection task such as (Monperrus et al., 2010). The second criterion is related to pattern ordering, where some approaches mine ordered patterns like (Montandon et al., 2013; Wang et al., 2013), while other ones mine unordered patterns such as (Monperrus et al., 2010; Bruch et al., 2006). The third one concerns the granularity of the elements composing patterns. For examples, in (Montandon et al., 2013; Wang et al., 2013), the approaches mine patterns composed of methods, and the approach in (Bruch et al., 2006) mines patterns composed of classes. The fourth one related to the technique that is used to identify the patterns. The used technique can be association rules mining like (Bruch et al., 2006), clustering algorithms such as (Wang et al., 2013) or a heuristic defined by the authors such as (Montandon et al., 2013; Monperrus et al., 2010). Some approaches combine many techniques, e.g., Unddin et al. used Principle Component Analysis with clustering algorithm (Uddin et al., 2012), and Buse and Weimer combined the clustering algorithm with their own proposed heuristic (Buse and Weimer, 2012).

\section{Conclusion and future work}

\subsection{Conclusion}

In this paper, we presented an approach that aims to mine software components from object-oriented APIs. This is based on static analysis of the source code of both the APIs and their software clients, in order to analyze the way that the software clients have used the API classes. The component identification process is use-driven. It implies that components are identified starting from classes composing their interfaces. Classes composing the provided interface of the first layer components compose FUPs. Then, the API is organized by a set of layers, where each layer composes of components providing services to the others composing the above layer, and so on.

The presented approach is experimented via four different APIs. These can be classified in terms of their size into medium (i.e., app, view and java.util), and large (i.e., android) and in terms of the implemented functionalities into extensive (i.e., java.util android) and specific (i.e., view and app). The validation is done through three research questions. The first one is related to the understandability, while the second indicates to the reusability. The results show that our approach improves the reusability and the understandability of the API. The third research question aims at compare our approach with a traditional component identification approach. The results prove that our approach outperforms the traditional one.

\subsection{Future work}

There are many future directions that are indicated by this research. These include:

1. Migrating the identified object-oriented components into existing component models. Components are identified as clusters of object-oriented classes representing their implementation. This constitutes the first step of the reengineering process of object-oriented software into componentbased software. Thus, we plan to extend our approach by transforming the object-oriented implementation of the identified components into an equivalent component-based one, such as OSGi (Tavares and Valente, 2008) and Fractal (Bruneton et al., 2006). We start by solving instantiation and inheritance transformation problems in (Alshara et al., 2015). We plan to investigate how to cope with other objectoriented dependencies between components, exception handling and component instantiation.

2. Identifying components based on dynamic analysis. To address static analysis limitations (dynamic binding/polymorphism), we plan to extend our approach through integrating a dynamic analysis technique.

3. Developing a visual environment. The presented approach can be extended by providing a visual environment, such that domain experts can supervise the approach steps and modify the obtained results when needed.

4. Experimenting with large number of case studies. The selection of API client applications affects the resulted component-based API. Thus, we plan to extend the evaluation of the proposed approach by conducting more case studies in order to further test the approach and to generalize the results as well.

5. Validating our approach by human experts. The results of the presented approach are validated based on heuristic 
measurements that we proposed. To better validate our approach, we plan to validate the results using the help of human experts.

\section{References}

Acharya, M., Xie, T., Pei, J., Xu, J., 2007. Mining api patterns as partial orders from source code: from usage scenarios to specifications. In: Proceedings of the 6th joint meeting of the European software engineering conference and the ACM SIGSOFT symposium on The foundations of software engineering. ACM, pp. 25-34.

Allier, S., Sadou, S., Sahraoui, H., Fleurquin, R., 2011. From object-oriented applications to component-oriented applications via component-oriented architecture. In: 2011 9th Working IEEE/IFIP Conf. on Software Architecture (WICSA). IEEE, pp. 214-223.

Alshara, Z., Seriai, A.-D., Tibermacine, C., Bouziane, H.L., Dony, C., Shatnawi, A., 2015. Migrating large object-oriented applications into component-based ones: instantiation and inheritance transformation. In: Proceedings of the 2015 ACM SIGPLAN International Conference on Generative Programming: Concepts and Experiences. ACM, pp. 55-64.

Antoniol, G., Guéhéneuc, Y.-G., 2005. Feature identification: a novel approach and a case study. In: Software Maintenance, 2005. ICSM'05. Proceedings of the 21st IEEE International Conference on. IEEE, pp. 357-366.

Benlarbi, S., Melo, W.L., 1999. Polymorphism measures for early risk prediction. In: Software Engineering, 1999. Proceedings of the 1999 International Conference on. IEEE, pp. 334-344

Bieman, J.M., Kang, B.-K., 1995. Cohesion and reuse in an object-oriented system. In: Proc. of the 1995 Symposium on Software Reusability. ACM, New York, NY USA, pp. 259-262. doi:10.1145/211782.211856.

Bruch, M., Schäfer, T., Mezini, M., 2006. Fruit: Ide support for framework understanding. In: Proc. of the 2006 OOPSLA Workshop on Eclipse Technology eXchange. ACM, New York, NY, USA, pp. 55-59. doi:10.1145/1188835.1188847.

Bruneton, E., Coupaye, T., Leclercq, M., Quema, V., Stefani, J.-B., 2006. The fractal component model and its support in java. Software 36 (11-12), 1257-1284. doi:10.1002/spe.767.

Buse, R.P.L., Weimer, W., 2012. Synthesizing api usage examples. In: Proc. of the 2012 Inter. Conf. on Software Engineering. IEEE Press, Piscataway, NJ, USA, pp. 782-792.

Chardigny, S., Seriai, A., Oussalah, M., Tamzalit, D., 2008. Extraction of componentbased architecture from object-oriented systems. In: Seventh Working IEEE/IFIP Conf. on Software Architecture (WICSA), pp. 285-288. doi:10.1109/WICSA.2008. 44.

Chardigny, S., Seriai, A.-D., Oussalah, M., Tamzalit, D., 2008. Search-based extraction of component-based architecture from object-oriented systems. In: 2nd European Conf. in Software Architecture (ECSA). In: Lecture Notes in Computer Science, 5292. Springer Berlin Heidelberg, pp. 322-325. doi:10.1007/ 978-3-540-88030-1_28.

Chen, K., Rajlich, V., 2000. Case study of feature location using dependence graph. In: In Proceedings of the 8th International Workshop on Program Comprehension.

Damaševičius, R., Paškevičius, P., Karčiauskas, E., Marcinkevičius, R., 2012. Automatic extraction of features and generation of feature models from java programs. Inf. Technol. Control 41 (4), 376-384

von Detten, M., Platenius, M.C., Becker, S., 2013. Reengineering component-based software systems with archimetrix. Softw. Syst. Model. 13 (4), 1-30.

Dit, B., Revelle, M., Gethers, M., Poshyvanyk, D., 2013. Feature location in source code: a taxonomy and survey. J. Softw. 25 (1), 53-95.

Ducasse, S., Pollet, D., 2009. Software architecture reconstruction: a process-oriented taxonomy. Softw. Eng., IEEE T. 35 (4), 573-591.

Duszynski, S., Knodel, J., Becker, M., 2011. Analyzing the source code of multiple software variants for reuse potential. In: Proc. of WCRE. IEEE, pp. 303-307.

Erdemir, U., Tekin, U., Buzluca, F., 2011. Object oriented software clustering based on community structure. In: 2011 18th Asia Pacific Software Engineering Conference (APSEC). IEEE, pp. 315-321.

Frakes, W., Kang, K., 2005. Software reuse research: status and future. IEEE T. Softw. Eng. 31 (7), 529-536. doi:10.1109/TSE.2005.85.

Garcia, J., Ivkovic, I., Medvidovic, N., 2013. A comparative analysis of software architecture recovery techniques. In: IEEE/ACM 28th Inter. Conf. on Automated Software Engineering (ASE), pp. 486-496. doi:10.1109/ASE.2013.6693106.

Google, 2015. API guides, (http://developer.android.com/reference/packages.html).

Han, J., Kamber, M., Pei, J., 2006. Data mining: concepts and techniques. Morgan Kaufmann.
Han, J., Pei, J., Yin, Y., 2000. Mining frequent patterns without candidate generation. In: ACM SIGMOD Record, 29. ACM, pp. 1-12.

ISO, 2001. Software Engineering - Product Quality - Part 1: Quality Model. Technical Report, ISO/IEC 9126-1. International Organization for Standardization.

Kang, K.C., Cohen, S.G., Hess, J.A., Novak, W.E., Peterson, A.S., 1990. Feature-oriented domain analysis (FODA) feasibility study. Technical Report. DTIC Document.

Kebir, S., Seriai, A.-D., Chardigny, S., Chaoui, A., 2012. Quality-centric approach for software component identification from object-oriented code. In: Joint Working IEEE/IFIP Conf. and European Conf. on Software Architecture (WICSA)/(ECSA), 2012, pp. 181-190. doi:10.1109/WICSA-ECSA.212.26.

Ma, H., Amor, R., Tempero, E., 2006. Usage patterns of the java standard api. In: 13th Asia Pacific Software Engineering Conf. APSEC 2006, pp. 342-352. doi:10.1109/ APSEC.2006.60.

Maalej, W., Robillard, M., 2013. Patterns of knowledge in api reference documentation. IEEE T. Softw. Eng. 39 (9), 1264-1282. doi:10.1109/TSE.2013.12.

Mishra, S., Kushwaha, D.S., Misra, A.K., 2009. Creating reusable software component from object-oriented legacy system through reverse engineering. J. Object Technol. 8 (5), 133-152.

Monperrus, M., Bruch, M., Mezini, M., 2010. Detecting missing method calls in object-oriented software. In: European Conf. on Object-Oriented Programming ECOOP. In: Lecture Notes in Computer Science, 6183. Springer Berlin Heidelberg pp. 2-25. doi:10.1007/978-3-642-14107-2_2.

Monperrus, M., Eichberg, M., Tekes, E., Mezini, M., 2012. What should developers be aware of an empirical study on the directives of api documentation. Emp. Softw. Eng. 17 (6), 703-737. doi:10.1007/s10664-011-9186-4.

Montandon, J., Borges, H., Felix, D., Valente, M., 2013. Documenting apis with examples: Lessons learned with the apiminer platform. In: 20th Working Conf. on Reverse Engineering (WCRE), pp. 401-408. doi:10.1109/WCRE.2013.6671315.

Poshyvanyk, D., Marcus, A., 2006. The conceptual coupling metrics for objectoriented systems. In: 22nd IEEE Inter. Conf. on Software Maintenance (ICSM) 2006, pp. 469-478, doi:10.1109/ICSM.2006.67.

Robillard, M., Bodden, E., Kawrykow, D., Mezini, M., Ratchford, T., 2013. Automated api property inference techniques. IEEE T. Softw. Eng. 39 (5), 613-637. doi:10. 1109/TSE.2012.63.

Shatnawi, A., Seriai, A., Sahraoui, H.A., Al-Shara, Z., 2015. Mining software components from object-oriented apis. In: Software Reuse for Dynamic Systems in the Cloud and Beyond - 14th International Conference on Software Reuse, ICSR 2015, Miami, FL, USA, January 4-6, 2015. Proceedings, pp. 330-347. doi:10.1007/ 978-3-319-14130-5_23.

Shatnawi, A., Seriai, A.-D., 2013. Mining reusable software components from objectoriented source code of a set of similar software. In: IEEE 14th Inter. Conf on Information Reuse and Integration (IRI), pp. 193-200. doi:10.1109/IRI.2013. 6642472.

Szyperski, C., 2002. Component Software: Beyond Object-Oriented Programming. Pearson Education.

Tavares, A.L.C., Valente, M.T., 2008. A gentle introduction to osgi. SIGSOFT Softw. Eng. Notes 33 (5), 8:1-8:5. doi:10.1145/1402521.1402526.

Uddin, G., Dagenais, B., Robillard, M.P., 2012. Temporal analysis of api usage concepts. In: Proc. of the 2012 Inter. Conf. on Software Engineering. IEEE Press, Piscataway, NJ, USA, pp. 804-814.

Wang, J., Dang, Y., Zhang, H., Chen, K., Xie, T., Zhang, D., 2013. Mining succinct and high-coverage api usage patterns from source code. In: Proc. of the 10th Working Conf. on Mining Software Repositories. IEEE Press, Piscataway, NJ, USA, pp. 319-328.

Weinreich, R., Miesbauer, C., Buchgeher, G., Kriechbaum, T., 2012. Extracting and facilitating architecture in service-oriented software systems. In: Joint Working IEEE/IFIP Conf. on Software Architecture (WICSA) and European Conf. on Software Architecture (ECSA), pp. 81-90. doi:10.1109/WICSA-ECSA.212.16.

Xue, Y., 2011. Reengineering legacy software products into software product line based on automatic variability analysis. In: Proceedings of the 33rd International Conference on Software Engineering. ACM, pp. 1114-1117.

Zhang, C., Yang, J., Zhang, Y., Fan, J., Zhang, X., Zhao, J., Ou, P., 2012. Automatic parameter recommendation for practical api usage. In: Proceedings of the 34th International Conference on Software Engineering. IEEE Press, pp. 826-836.

Ziadi, T., Frias, L., da Silva, M.A.A., Ziane, M., 2012. Feature identification from the source code of product variants. In: Software Maintenance and Reengineering (CSMR), 2012 16th European Conference on. IEEE, pp. 417-422.

Zibran, M., Eishita, F., Roy, C., 2011. Useful, but usable factors affecting the usability of apis. In: 18th Working Conf. on Reverse Engineering (WCRE), pp. 151-155. doi:10.1109/WCRE.2011.26. 


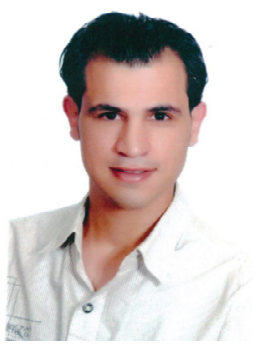

Anas Shatnawi is a post-doctoral researcher at the laboratory for research on technology for ecommerce (LATECE) at University of Quebec at Montreal, Canada. He has a PhD degree from University of Montpellier, France. He obtained a M.Sc. in Computer Science from the Jordon University of Science and Technology in 2012, Jordan. His primarily interest is in software engineering with a particular focus on reengineering, reverse engineering, APIs reusability, software architectures, components, services and software product lines.

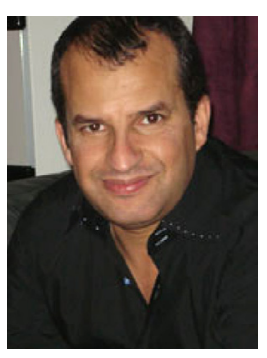

Abdelhak-Djamel Seriai is associate professor at University of Montpellier. He obtained his engineer degree in 1994. He obtained his PhD degree from University of Nantes, France in 2001. His research interests include software reuse, software architecture, software reengineering, reverse engineering, software component/service, software product line, software evolution, source code analysis, search-based algorithms, etc. He is author or co-author of more than 50 publications in international journals and conferences. He is the scientific editor of the first French book on "software evolution and maintenance". He is owner of the French scientific excellence reward from 2009 to 2013 and from 2014 to 2018 (award given by the French government in recognition of the scientific quality of a researcher).

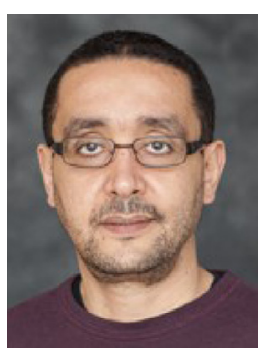

Houari A. Sahraoui is professor at the department of computer science and operations research (GEODES, software engineering group) of University of Montreal. His research interests include software engineering automation, model-driven engineering, software visualization, and search-based software engineering. He has served as a program committee member in several IEEE and ACM conferences, as a member of the editorial boards of four journals, and as an organization member of many conferences and workshops.

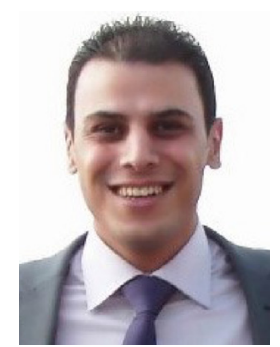

Zakarea Alshara is a PhD student at the laboratory of Informatics, Robotics and Microelectronics of Montpellier (LIRMM) at University of Montpellier, France. He obtained a M.Sc. in Computer Science from the Jordon University of Science and Technology in 2013, Jordan. His research interests are in software engineering and cloud computing with a focus on software analysis, reengineering, code transformation, software architecture, and component-based software engineering. 